\title{
Article \\ Contactless Vital Sign Monitoring System for Heart and Respiratory Rate Measurements with Motion Compensation Using a Near-Infrared Time-of-Flight Camera
}

\author{
Kaiwen Guo ${ }^{1, *}$, Tianqu Zhai ${ }^{1}\left(\mathbb{D}\right.$, Elton Pashollari ${ }^{1}$, Christopher J. Varlamos ${ }^{\mathbb{1}}{ }^{2}$, Aymaan Ahmed ${ }^{3}$ \\ and Mohammed N. Islam ${ }^{1,4}$ \\ 1 Department of Electrical Engineering, University of Michigan, Ann Arbor, MI 48109, USA; \\ tianquzh@umich.edu (T.Z.); epasholl@umich.edu (E.P.); mni@eecs.umich.edu (M.N.I.) \\ 2 School of Medicine, Case Western Reserve University, Cleveland, OH 44106, USA; cxv140@case.edu \\ 3 Department of Computer Science and Engineering, University of California, La Jolla, \\ San Diego, CA 92093, USA; aaa044@ucsd.edu \\ 4 Omni Science Inc., Dexter, MI 48130, USA \\ * Correspondence: kaiwguo@umich.edu
}

check for updates

Citation: Guo, K.; Zhai, T.;

Pashollari, E.; Varlamos, C.J.; Ahmed,

A.; Islam, M.N. Contactless Vital Sign

Monitoring System for Heart and

Respiratory Rate Measurements with

Motion Compensation Using a

Near-Infrared Time-of-Flight Camera.

Appl. Sci. 2021, 11, 10913. https://

doi.org/10.3390/app112210913

Academic Editor: Paolo Visconti

Received: 14 October 2021

Accepted: 16 November 2021

Published: 18 November 2021

Publisher's Note: MDPI stays neutral with regard to jurisdictional claims in published maps and institutional affiliations.

Copyright: (c) 2021 by the authors. Licensee MDPI, Basel, Switzerland. This article is an open access article distributed under the terms and conditions of the Creative Commons Attribution (CC BY) license (https:/ / creativecommons.org/licenses/by/ $4.0 /)$.

\begin{abstract}
This study describes a contactless vital sign monitoring (CVSM) system capable of measuring heart rate (HR) and respiration rate (RR) using a low-power, indirect time-of-flight (ToF) camera. The system takes advantage of both the active infrared illumination as well as the additional depth information from the ToF camera to compensate for the motion-induced artifacts during the HR measurements. The depth information captures how the user is moving with respect to the camera and, therefore, can be used to differentiate where the intensity change in the raw signal is from the underlying heartbeat or motion. Moreover, from the depth information, the system can acquire respiration rate by directly measuring the motion of the chest wall during breathing. We also conducted a pilot human study using this system with 29 participants of different demographics such as age, gender, and skin color. Our study shows that with depth-based motion compensation, the success rate (system measurement within $10 \%$ of reference) of HR measurements increases to $75 \%$, as compared to $35 \%$ when motion compensation is not used. The mean HR deviation from the reference also drops from $21 \mathrm{BPM}$ to $-6.25 \mathrm{BPM}$ when we apply the depth-based motion compensation. In terms of the RR measurement, our system shows a mean deviation of 1.7 BPM from the reference measurement. The pilot human study shows the system performance is independent of skin color but weakly dependent on gender and age.
\end{abstract}

Keywords: time-of-flight; contactless physiological measurement; heart rate monitoring; motion artifacts compensation

\section{Introduction}

We demonstrate a contactless vital sign monitoring (CVSM) system based on a nearinfrared indirect time-of-flight (ToF) camera that can measure the heart rate (HR) and the respiratory rate (RR) of an individual at a stand-off distance. The system utilizes a vertical-cavity surface-emitting laser (VCSEL) illuminated indirect ToF camera, which measures both grayscale intensity and depth information simultaneously. We use grayscale intensity information as well as the distance information from two regions of interest (ROI) of a participant to determine the HR and RR. The HR is derived from periodic intensity change on a participant's face induced by blood flow from the heartbeats, while the RR is determined by measuring the periodic change of chest wall movement during breathing. We find that the motion artifacts can dominate the underlying HR signal especially when an active illumination source such as VCSEL lasers are used. In this study, we use the depth information from the ToF camera to compensate for these motion artifacts since the 
depth information can be used to differentiate the grayscale intensity variations caused by underlying heartbeat versus the user's motion.

We conducted a pilot human study with 29 participants of varying demographics to show that when using distance-based motion compensation, the success rate of HR measurement increases to $75 \%$, as compared to only $35 \%$ when distance-based motion compensation is not used. The mean deviation from the reference HR measurement is $-6.25 \mathrm{BPM}$ if we apply the depth signal for motion compensation, while the deviation increases to $21 \mathrm{BPM}$ when motion compensation is not used. At the same time, our study shows that the mean deviation of our RR measurement is 1.7 BPM, which is comparable to the pulse oximeter we used in this study. Our results further show that the system performs well among participants of various demographics such as skin color, gender, and age. Such systems could be useful in applications such as vehicle driver/occupant monitoring systems, remote and in-patient patient monitoring systems, telemedicine systems, or unobtrusive health screening systems.

\section{Background and Motivation}

Contactless physiological monitoring systems working at a remote distance have attracted much attention over the past decades. Especially after the COVID-19 pandemic in early 2020, there has been an increased demand for contactless physiological monitoring systems for health monitoring/screening at a safe distance to reduce the spread of viruses. Out of many potential physiological parameters, heart rate and respiration rate have attracted much attention. Table 1 briefly summarizes the characteristics of various approaches in monitoring $\mathrm{HR} / \mathrm{RR}$. To date, most contactless $\mathrm{HR} / \mathrm{RR}$ measurement systems rely on photoplethysmography (PPG) using RGB (red-green-blue) cameras with visible light illumination [1-8]. Several approaches, such as the skin-tone or blind-source separation-based algorithm, are proposed for the removal of external artifacts and considerably improve the reliability of HR measurement [9-16]. However, in some application scenarios such as vehicle driver monitoring or patient monitoring in hospitals, RGB camerabased systems face challenges from fluctuating or very low background illumination [17]. Moreover, because melanin, which gives rise to skin color, has $\sim 5 \mathrm{X}$ higher absorption in visible wavelength of $530 \mathrm{~nm}$ as compared to $850 \mathrm{~nm}$ [18], the performance of RGB camera-based systems can vary among users of different skin colors $[6,10,17]$. By using near-infrared illumination, such skin color dependence can be mitigated to achieve more uniform performance among wider demographics. Due to the benefits of being independent of background illumination and users' skin color, remote HR and RR monitoring systems using a near-infrared light source can be a valuable alternative.

Table 1. Comparison of HR measuring devices.

\begin{tabular}{|c|c|c|c|c|c|}
\hline System & $\begin{array}{c}\text { Signal } \\
\text { Strength }\end{array}$ & Contactless & $\begin{array}{c}\text { Skin Color } \\
\text { Independent }\end{array}$ & $\begin{array}{l}\text { Ambient Light } \\
\text { Independent }\end{array}$ & $\begin{array}{c}\text { Direct }{ }^{*} \mathrm{RR} \\
\text { Measurement }\end{array}$ \\
\hline Wearable EKG (Polar H10 belt) & ++ & $\boldsymbol{x}$ & $\checkmark$ & $\checkmark$ & $\boldsymbol{*}$ \\
\hline Pulse Oximete (Masimo Mightysat & + & $\boldsymbol{*}$ & $\boldsymbol{*}$ & & $\boldsymbol{*}$ \\
\hline RGB Camera [1-8] & $=$ & $\gamma$ & * & $\boldsymbol{x}$ & $\boldsymbol{x}$ \\
\hline Infrared Camera [19-23] & - & $\checkmark$ & $\checkmark$ & $\checkmark$ & * \\
\hline ToF Camera [24], [This] & - & $\gamma$ & $\checkmark$ & $\checkmark$ & $\checkmark$ \\
\hline Doppler Radar [25] & - & $\checkmark$ & $\checkmark$ & $\checkmark$ & $\checkmark$ \\
\hline
\end{tabular}

${ }^{*}$ A direct measurement measures chest movement meanwhile an indirect RR measurement uses the modulation of the HR signal to extract RR [26,27];+/=/- indicates system has advantage/neutral/disadvantage in certain category; $/ / \boldsymbol{*}$ indicates system has certain capability.

Over the past few years, HR/RR measurement systems using near-infrared cameras or even RGB/Depth cameras (such as Microsoft Kinect) [19-24,28,29] have been studied to show their capability of measuring HR/RR. In this study, we propose a system that 
leverages the existing ToF cameras in many consumer electronics and vehicles to perform HR and RR monitoring. The ToF camera used in this study is an indirect ToF using a modulated VCSEL laser as its light source [30]. The benefit of this type of ToF camera is that it can extract the grayscale and depth information at the same time. The grayscale image is directly acquired through the exposure of the imaging sensor while the depth image is acquired by examining the phase change of the returned light [30]. Compared to previous studies, one of the main advantages and technical advancements of this study is that we demonstrate a method that combines both the depth and grayscale intensity information from the ToF camera to compensate for motion artifacts. Motion artifacts are known to be a challenging issue for contactless PPG systems, but even more so for systems that operate in the near-infrared wavelengths because the absorption by hemoglobin is much weaker in the near-infrared compared to visible wavelengths $[31,32]$. As a result, the PPG signal strength between $850 \mathrm{~nm}$ to $1000 \mathrm{~nm}$ is only about $1 / 8$ th the peak amplitude at $530 \mathrm{~nm}$. Since the ToF camera directly measures the body motion of a participant, we find this information useful in distinguishing intensity changes caused by body motion versus heartbeat, which would allow us to compensate for motion-induced artifacts. In addition, by measuring the motion of the chest wall using the ToF camera, we can at the same time directly measure the RR of a participant, instead of extracting RR from HR or other indirect body motion when an RGB camera is used [26,27]. Furthermore, to our knowledge, this is the first study that investigates the performance of a ToF-based HR/RR monitor system over larger and diverse demographics [32]. A more detailed comparison between this study and previous studies using IR cameras is presented in Table 2. Since indirect ToF cameras are being widely used in many consumer electronics, our CVSM system would enable low-cost, reliable, and multi-modality physiological signal monitoring on various platforms and application scenarios in the future. For example, such a system can be mounted in a vehicle to help monitor the physiological condition of the driver in a continuous manner. If any unexpected events such as falling asleep or heart attack were to happen, the CVSM system can then alert the autonomous/assisted driving system of the vehicle to bring the vehicle to a safe stop.

Table 2. Summary of recent studies on IR camera-based HR/RR monitoring system.

\begin{tabular}{cccccc}
\hline Reference & $\begin{array}{c}\text { Wavelength } \\
(\mathbf{n m})\end{array}$ & $\begin{array}{c}\text { Camera } \\
\text { Type }\end{array}$ & $\begin{array}{c}\text { Avg. HR } \\
\text { Error (BPM) }\end{array}$ & $\begin{array}{c}\text { Measures } \\
\text { RR? }\end{array}$ & $\begin{array}{c}\text { Avg. RR } \\
\text { Error (BPM) }\end{array}$ \\
\hline$[19]$ & $675 / 800 / 842$ & NIR & 0.76 & No & N/A \\
{$[21]$} & 940 & NIR & 13.6 & No & N/A \\
{$[24]$} & 850 & ToF & 3.46 & No & N/A \\
{$[29]$} & 850 & NIR & 2 & No & N/A \\
This & 850 & ToF & 6.25 & Yes & 1.7 \\
\hline
\end{tabular}

\section{Experiment Setup}

The indirect ToF camera used in this study is a CamBoard Pico Flexx camera (PMD Technologies AG, Siegen, Germany). This camera contains a 3D CMOS sensor with a resolution of $171 \times 224$, and a field of view of $62^{\circ} \times 45^{\circ}$. The illumination source on the camera is an 850nm VCSEL with eye-safe power output and an operating distance from 0.1 to $4 \mathrm{~m}$. This ToF camera comes with a compact form factor of $68 \mathrm{~mm} \times 17 \mathrm{~mm} \times 7.35 \mathrm{~mm}$ and consumes only $300 \mathrm{~mW}$ average power, which makes it ideal for mobile application scenarios. In our experiment, the ToF camera is connected via USB to a laptop (2019 Lenovo X1 Extreme), which is used to both control the camera and process the recorded $3 \mathrm{D}$ video to extract $\mathrm{HR}$ and $\mathrm{RR}$. The ToF camera is mounted on a stand positioned at the same height and $\sim 60 \mathrm{~cm}$ away from the participants, which allows us to capture a participant's face and chest region in one frame (Figure 1). To provide a reference HR and $\mathrm{RR}$ reading, a contact-based finger pulse oximeter capable of recording both $\mathrm{HR}$ and RR with accuracy down to +/ - 1 BPM (Mighty Sat Rx, Masimo, Irvine, CA, USA) is placed on 
one of the participant's index fingers. All measurements are made under the illumination of fluorescent light (mounted on the ceiling, powered by AC electricity at $60 \mathrm{~Hz}$ ), no special control of ambient light illumination is applied during the study.

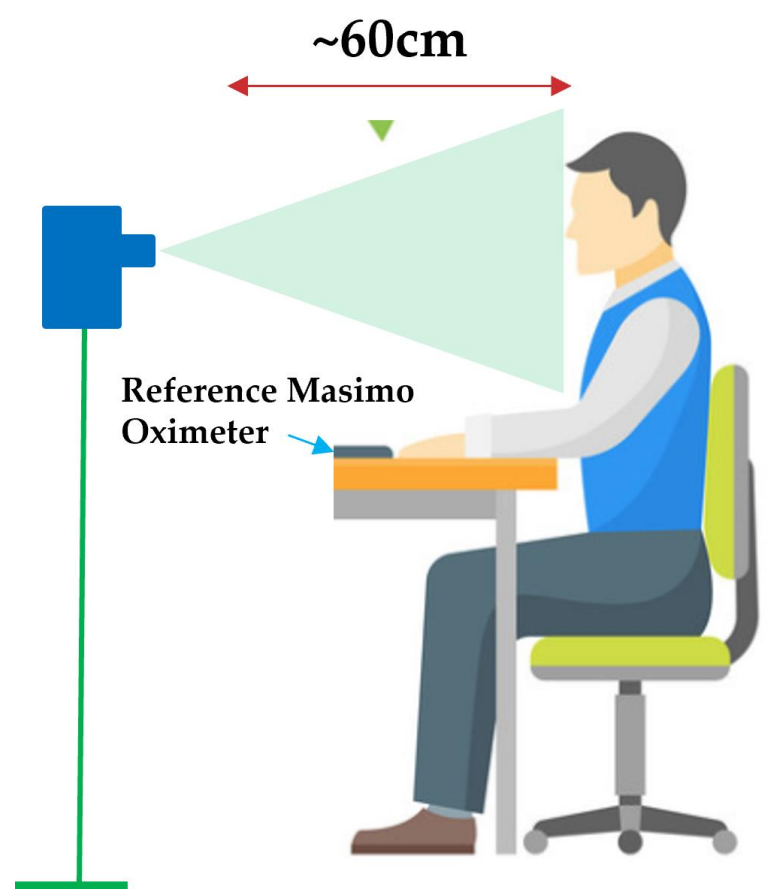

Figure 1. Experimental configuration for proof-of-concept $H R$, RR measurements using the Pico Flex indirect ToF camera.

To determine the HR and RR of a participant, two ROIs are used to extract HR and RR of the participant (Figure 2): ROI-1 is used for HR extraction while ROI-2 is used for RR extraction. The location of ROI- 1 that covers both the nose and cheek is calculated by evaluating the relative position between the participant's eyes and nose with a 66-point facial landmark detection algorithm [33]. The ROI-2 region that represents the upper chest regions is determined from the relative position between the jawline and the shoulder using a pose estimator [34]. Specifically, the system uses both grayscale intensity and depth information from ROI-1 to derive the HR while only the depth information from ROI-2 (upper chest) is used to derive the RR.

One advantage of using an indirect ToF camera for physiological monitoring is that it can operate under low or complex ambient light conditions. The enhanced immunity against external lighting conditions is a result of the narrow band-pass filter (centered at $850 \mathrm{~nm}$ ) placed in front of the optical lens as well as the background illumination suppression from the detection mechanism used in the indirect ToF camera [30]. To demonstrate the benefits of being independent of ambient lighting in physiological measurements, we show the results of HR measurement under various lighting conditions: a dark environment, a bright environment, and an environment where ambient light is switched on/off periodically. Figure 3 shows the (a) raw intensity from ROI- 1 when measured under the dark/bright environment and the (b) corresponding frequency domain information. When measuring under the dark environment, all ambient lights are switched off and the background illumination is reduced to $<1$ Lux. When measuring under the bright environment, in addition to the ambient fluorescent lighting, an $100 \mathrm{~W}$ halogen lamp is placed $1 \mathrm{~m}$ from the participant to create an illumination of 960 Lux. In both cases, since our system relies on the built-in VCSEL laser for illumination, a clear HR signal can still be seen in the time domain and the correct HR rate is obtained using a Fourier transform. 


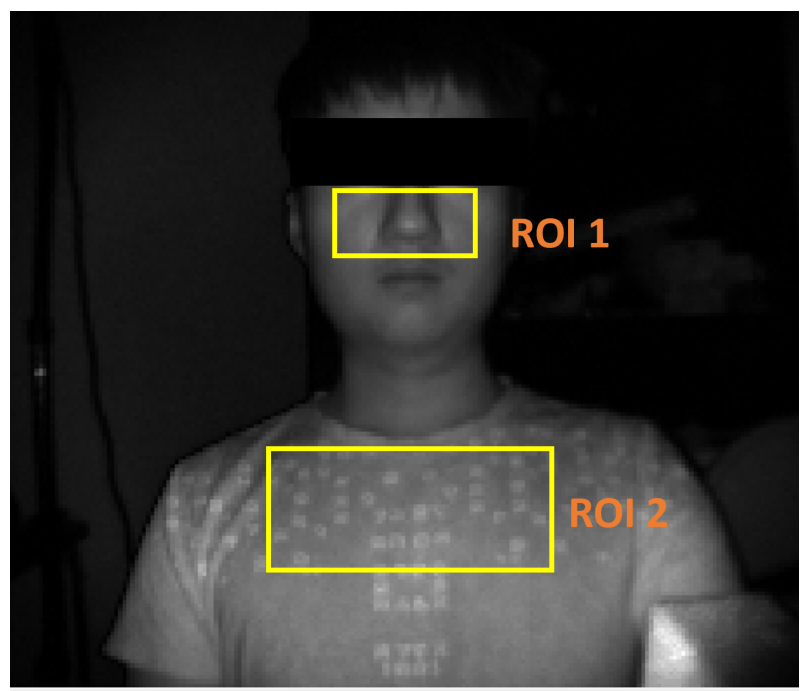

Figure 2. Two ROIs used for RR and HR extraction: ROI-1 is used for HR measurements while ROI-2 is used for RR measurements.
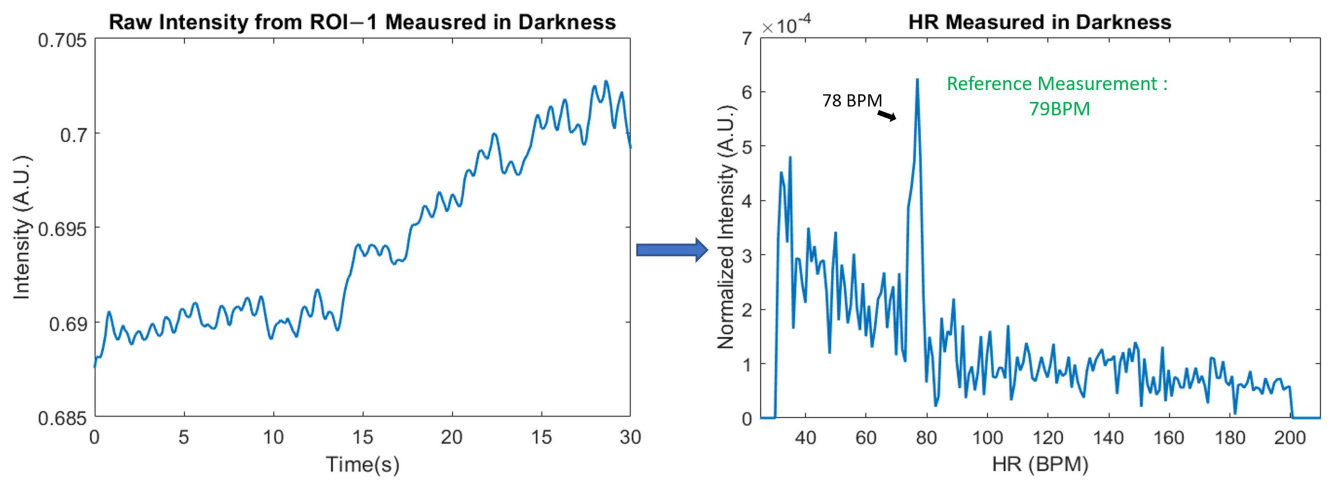

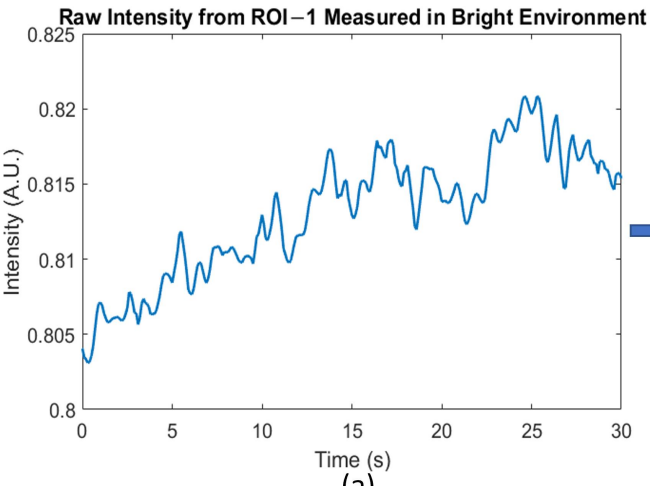

(a)

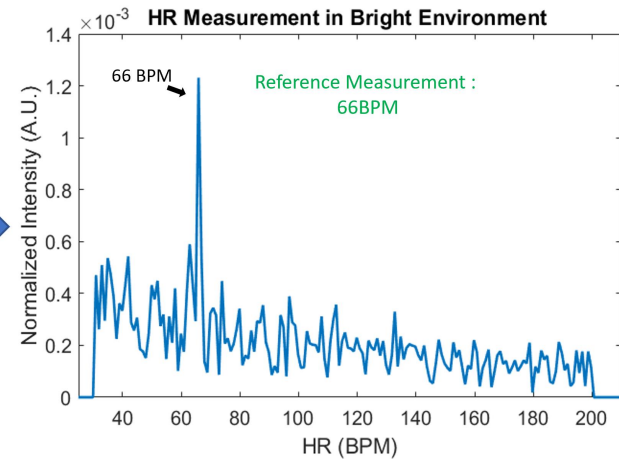

(b)

Figure 3. (a) Raw intensity signal from ROI-1 measured in dark/bright environment. The grayscale intensity shows a clear HR signal. (b) Frequency domain HR measured in dark/bright environment. The highest peak in the frequency domain correspond to measured HR.

We also demonstrate that the ToF camera can operate under more extreme lighting conditions. Figure 4 shows a measurement obtained with ambient light switched on and off at an interval of $5 \mathrm{~s}$. When the ambient light is turned off, the background illumination intensity is less than 1 Lux while when the ambient light is turned on, the background illumination is 330 Lux. No significant effects of ambient light being turned on/off are seen in Figure $4 \mathrm{a}$ and a correct HR measurement is still obtained with a Fourier transform. 


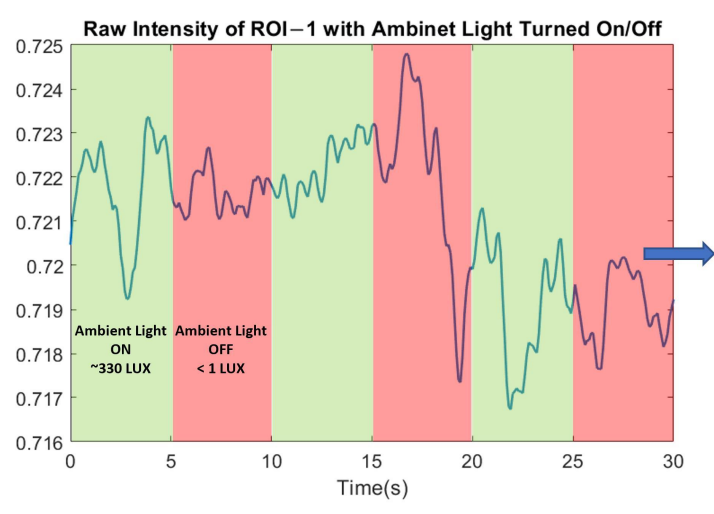

(a)

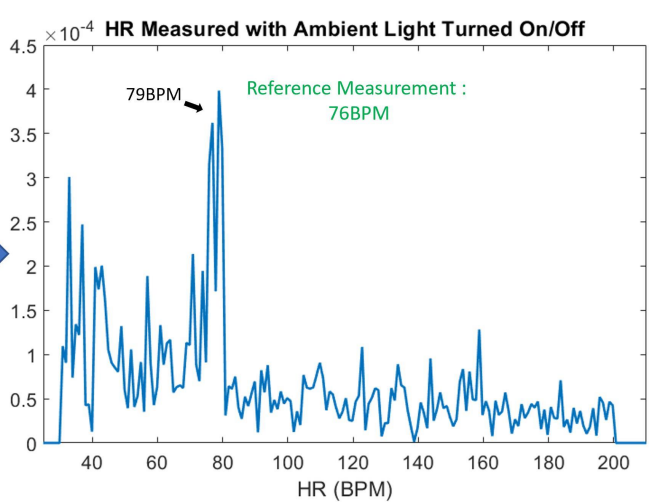

(b)

Figure 4. (a) Raw intensity signal from ROI-1 measured under alternating dark and bright environment. The ambient is swithced on/off at an interval of $5 \mathrm{~s}$. (b) Frequency domain HR measured under alternating lighting condition. The highest peak in the frequency domain correspond to measured HR.

Motion artifact is a key source of noise in contactless HR measurements, especially on a system with low power and active illumination such as the one in this study. When no motion or very limited motion is present, the HR can be obtained directly from the raw grayscale intensity from the ToF camera, as is shown in Figure 5a, during which the participant is asked to remain as still as possible. However, in more realistic scenarios, when the users are behaving naturally, there will inevitably be more motion artifacts present in the raw intensity data (Figure $5 b$ ). Such motion artifacts are especially obvious in our system where active illumination from a point source is used. Intensity changes caused by the user moving closer or further from the light source can be many times stronger than the underlying heartbeat signal, leading to erroneous HR measurements (Figure 5b).

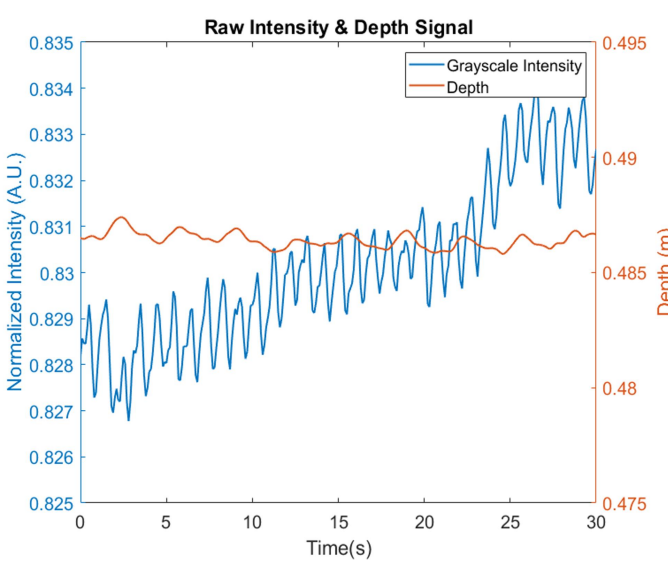

(a)

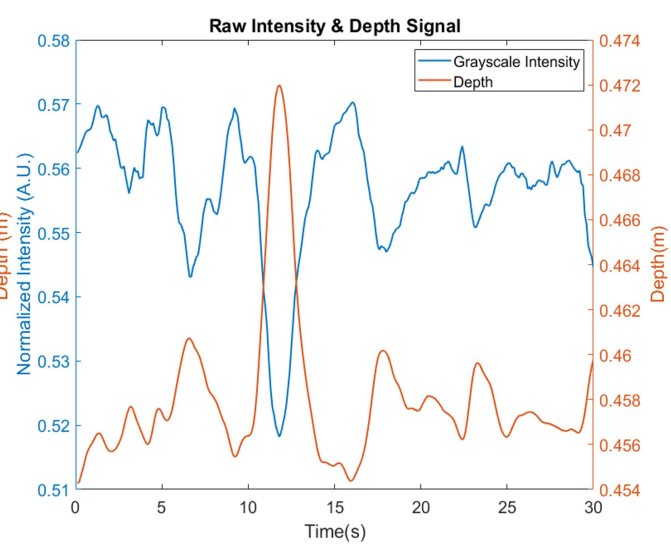

(b)

Figure 5. (a) Raw intensity signal (blue) and depth signal (red) from ROI-1 when participants are intentionally staying still. The grayscale intensity shows a clear HR signal. (b) Raw intensity signal (blue) and depth signal(red) from ROI-1 when motion artifact is present. The grayscale intensity is dominated by motion induced intensity artifacts.

Therefore, in this study, depth information is used to compensate for the intensity variation caused by motion artifacts. The underlying logic in the motion artifacts compensation is three-fold: First, because active illumination from a point source is used, the change of grayscale intensity is nonlinearly correlated to the relative position between the head and the camera. Secondly, the depth information recorded by the ToF camera should contain no heart rate component since the micromotion created by the heartbeat is too small to be measured by the ToF camera. Lastly, after the motion artifacts are removed, 
the compensated intensity information should have a minimum correlation with the depth information. In other words, the motion compensation is achieved when the relationship in Equation (1) is satisfied, where $I_{c o m p}, I_{\text {raw }}$, and $D_{\text {raw }}$ are compensated grayscale intensity, raw average intensity across all pixels in ROI-1, and raw average depth signal across all pixels in ROI-1 respectively. $a$ is the linear coefficient and $b$ is the nonlinear coefficient that minimize the correlation between $D_{\text {raw }}$ and $\frac{I_{\text {raw }}}{a *\left(D_{\text {raw }}\right)^{-b}}$

$$
I_{\text {comp }}=\frac{I_{\text {raw }}}{a *\left(D_{\text {raw }}\right)^{-b}} ; \text { where } a, b=\underset{a, b}{\operatorname{argmin}} \operatorname{Correlation}\left(\frac{I_{\text {raw }}}{a *\left(D_{\text {raw }}\right)^{-b}}, D_{\text {raw }}\right)
$$

Ideally, the intensity variation caused by the distance variation between the participants' faces and the light source should be inversely proportional to the square of the distance (i.e., inverse square law of light propagation). However, in reality, skin conditions as well as the illumination angle change from person to person $[35,36]$ and we found that different values of the nonlinear coefficients are needed to minimize the correlation between the $D_{\text {raw }}$ and $I_{\text {comp }}$ across different participants. In this study, the range of the nonlinear coefficient is set between 0.1 and 5 .

Based on the above logic, we compensate for the motion artifact and extract HR using the following steps (Figure 6): (a) ROI-1 is defined and tracked in all frames using a Kanade-Lucas-Tomasi feature tracker [37]; (b) the intensity across pixels within ROI-1 is averaged; (c) within a time window, using the relationship described in Equation (1), we calculate the compensated intensity $I_{\text {comp }}$ that has the minimum correlation between the depth signal $D_{\text {raw }}$ and $I_{\text {comp }}$; (d) a bandpass filter between 40 BPM and 150 BPM (which is the common resting HR range) is applied to the compensated signal $I_{\text {comp }}$; (e) a Fourier transform of the data is taken on the motion-compensated intensity signal $I_{\text {comp }}$ to find the HR, where HR is determined as the frequency component with the highest amplitude. In this study, we compare the performance of the motion-compensated HR results against the results that "do not use the motion compensation". The results that "do not use motion compensation" refer to HR reading derived using the same steps above except for the step of using the depth information to compensate for the motion artifacts.

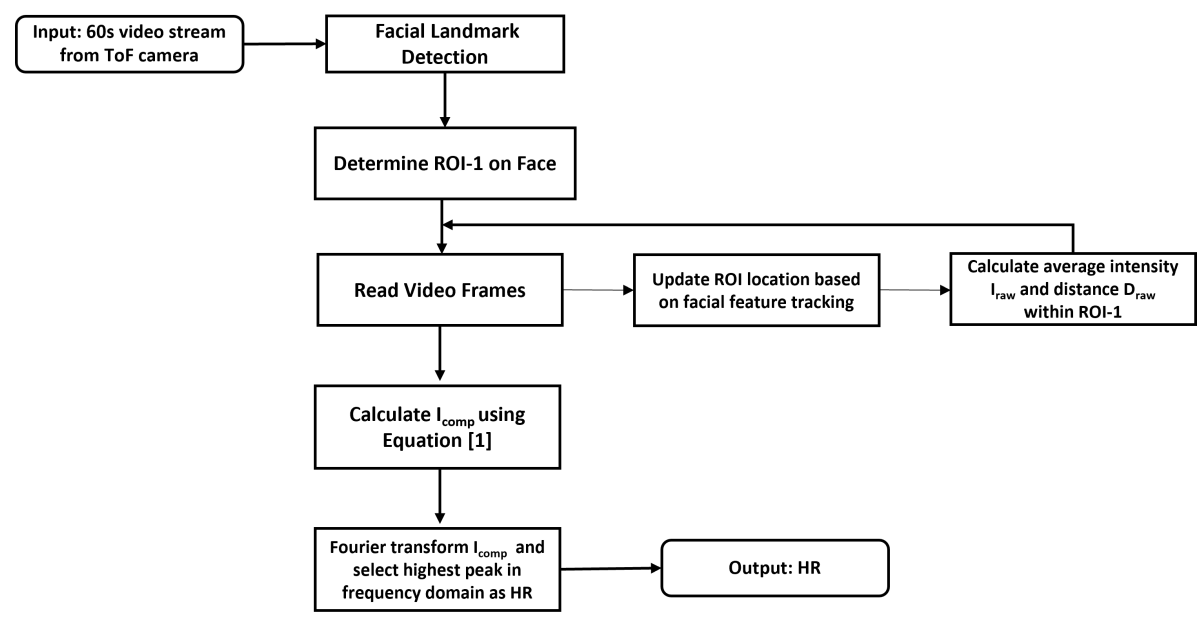

Figure 6. HR extraction process from the $3 \mathrm{D}$ video streams of a ToF camera.

RR extraction is more straightforward as we directly measure the chest wall movement from respiration using the depth signal from the chest region. Specifically, RR is extracted using the following steps (Figure 7): (a) ROI-2 is defined and tracked in all frames; (b) calculate the average depth across all pixels within ROI-2; (c) apply Fourier transform to the depth signal and apply bandpass filter between $5 \mathrm{BPM}$ to $30 \mathrm{BPM}$ to find the highest peak as the RR value. 


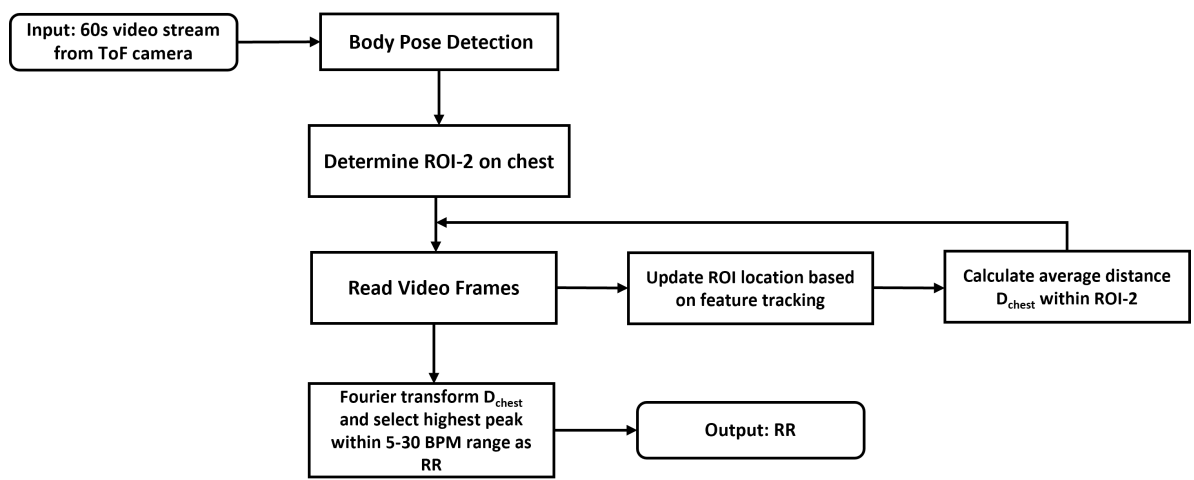

Figure 7. RR extraction process from the $3 \mathrm{D}$ video streams of a ToF camera.

To test the effectiveness of the HR/RR monitoring system, 29 participants from varying demographics were recruited in the study so that performance among different skin colors, genders, and ages could be compared. The study is approved by the Institutional Review Board at the University of Michigan and informed consent was obtained from every participant. All 29 participants were classified as either lighter skin color group or darker skin color group based on their Fitzpatrick skin type, a numerical classification for skin type based on a self-reported questionnaire [38]. Participants with skin type I-III were classified into the lighter skin color group, while participants with skin type IV-VI were classified into the darker skin color group. A total of 17 participants in this study were within the lighter skin color group, while the remaining 12 participants were considered as being within the darker skin color group. In terms of gender, 18 participants were male and 11 participants female. As for age, we recruited participants from 18 years old to 72 years old. All participants were divided into groups older than 35 years old and younger than 35 years old. In this study, 8 participants were older than 35 years old and the remaining 21 participants were younger than 35 years old. The demographics of the participants are shown in Table 3. A total of $1060 \mathrm{~s}$ videos at 10 frames per second are obtained from each participant. To mimic the real-world scenario, participants were asked to sit in front of the camera and are allowed to move as they see comfortable (such as leaning backward/forward) but they were asked to look toward the camera during the measurements. Among the 290 measurements, 4 measurements were excluded in the study due to the failure of the face recognition algorithm to identify the regions of interest (ROIs). In the end, 286 measurements were used in the study.

Table 3. Demographics of the participant population.

\begin{tabular}{|c|c|c|c|c|c|c|}
\hline Group: & $\begin{array}{l}\text { Number } \\
29\end{array}$ & of Participants & $\begin{array}{l}\text { Age Range } \\
18-72 \text { years }\end{array}$ & & $\begin{array}{l}\text { Skin } \\
\text { II-IV }\end{array}$ & Range \\
\hline Subgroup: & $\begin{array}{l}\text { (Female } \\
11\end{array}$ & $\begin{array}{l}\text { Male) } \\
18\end{array}$ & $\begin{array}{l}(<=35 \text { years } \\
21\end{array}$ & $\begin{array}{l}>35 \text { years }) \\
8\end{array}$ & $\begin{array}{l}(\mathrm{I}-\mathrm{III} \\
17\end{array}$ & $\begin{array}{l}\text { IV-VI) } \\
12\end{array}$ \\
\hline
\end{tabular}

\section{Experiment Results}

The raw and processed data from the two ROIs are illustrated in Figures 8 and 9. Figure 8 shows a $30 \mathrm{~s}$ segment of a typical $60 \mathrm{~s}$ recording of both the raw intensity and depth signal for ROI-1. The second row corresponds to the raw intensity information and the bottom row corresponds to the depth information from the same ROI-1. In ROI-1, the maximum motion from ROI-1 to the camera is as large as $15 \mathrm{~cm}$. As a result, the raw intensity data is strongly inversely correlated to the depth information from the same ROI, suggesting that the raw signal is dominated by the motion artifacts. The grayscale intensity after motion compensation is shown on the top row of Figure 8 where most of the motion artifacts in the depth information are removed. From the time-domain signal, the heart rate signal becomes recognizable compared to the motion-dominated raw signal. The effect 
of the motion artifacts as well as the benefits of depth-based motion compensation is also evident in the frequency domain. When comparing the frequency domain signal of the raw intensity and the depth signal, we can see the two spectra are similar to each other in shape, and they also share many common lower frequency components. Even though a weak frequency peak near the reference HR of 83 BPM can be seen, it is overshadowed by the frequency component from the motion artifacts. After the motion compensation, we can see that in the frequency domain signal of the compensated signal (Figure 8b, top), these artifact frequencies are suppressed and a clear HR frequency near 83 BPM can be seen in the compensated spectrum.

As for the respiration rate measurement, Figure 9 shows a typical depth signal from the ROI-2 (chest) region corresponding to a RR of $9 \mathrm{BPM}$. The amplitude of chest wall displacement caused by the respiration motion is approximately $4 \mathrm{~mm}$. Because of this small amplitude, such motion could be affected by other factors such as overall body motion as well the blockage of clothing. Some potential solutions to mitigate these issues will be discussed in the discussion section.
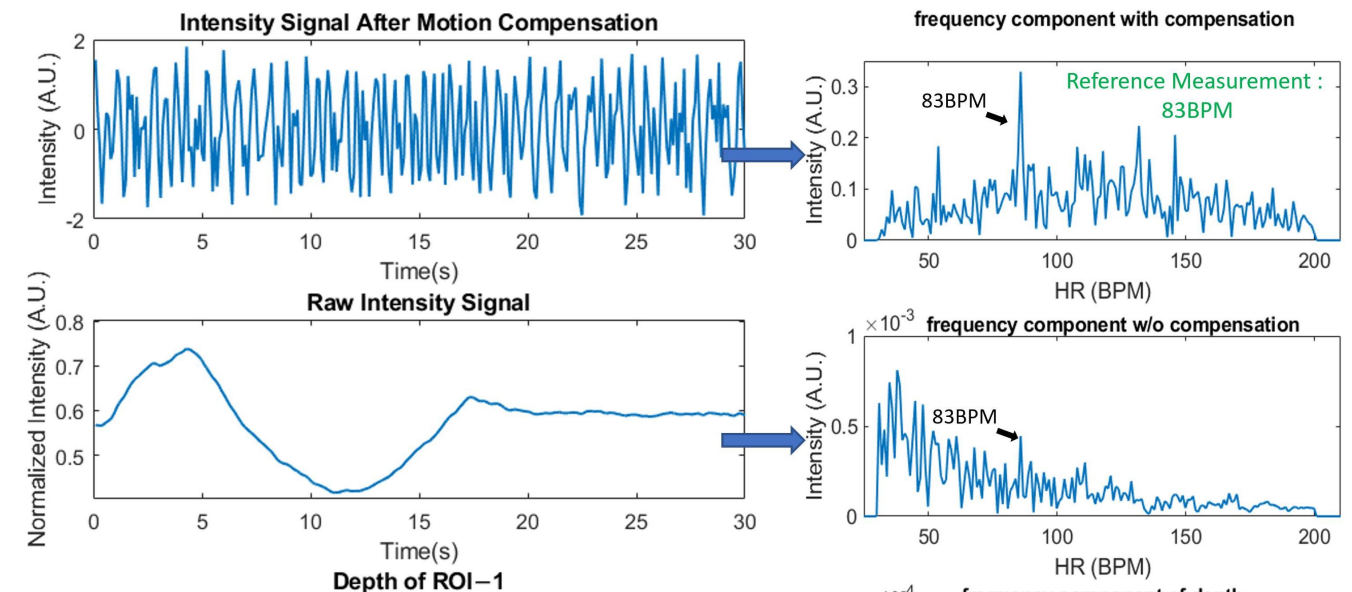

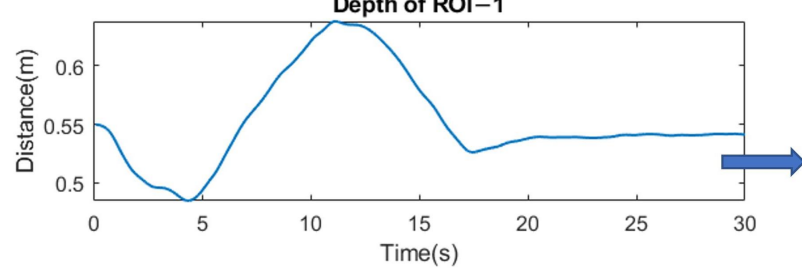

(a)

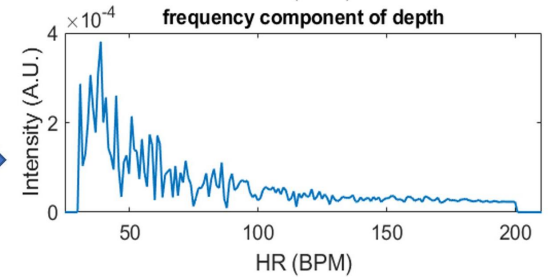

(b)

Figure 8. (a) Time domain signal for HR extraction: HR signal after motion compensation (top), raw grayscale signal (middle) and raw depth signal (bottom). (b) Frequency domain HR signal: with motion-compensation (top), without motion compensation (bottom).

The pilot human study with 29 participants illustrates the improvement in overall performance achieved for the HR measurements by using depth information compensation. In the study, the mean HR reading during the $60 \mathrm{~s}$ of measurement is considered as the ground truth and the "correct measurement" from our system is defined as a HR measurement that is within 10 percent of the ground truth. The top bar graph in Figure 10 demonstrates the distribution of measurement error rate after compensation, while the bottom bar graph demonstrates the distribution of measurement error rate without depth compensation. With depth information used to compensate for motion artifacts, $75 \%$ of the HR measurements (214 out of 286 measurements) are correct. On the other hand, without compensation, only $35 \%$ of the HR measurements (100 out of 286 tests) are correct. It is also worth noticing that with motion compensation, many of the erroneous measurements are below $30 \%$ error rate, as compared to between 50 and $60 \%$ for the uncompensated case. 


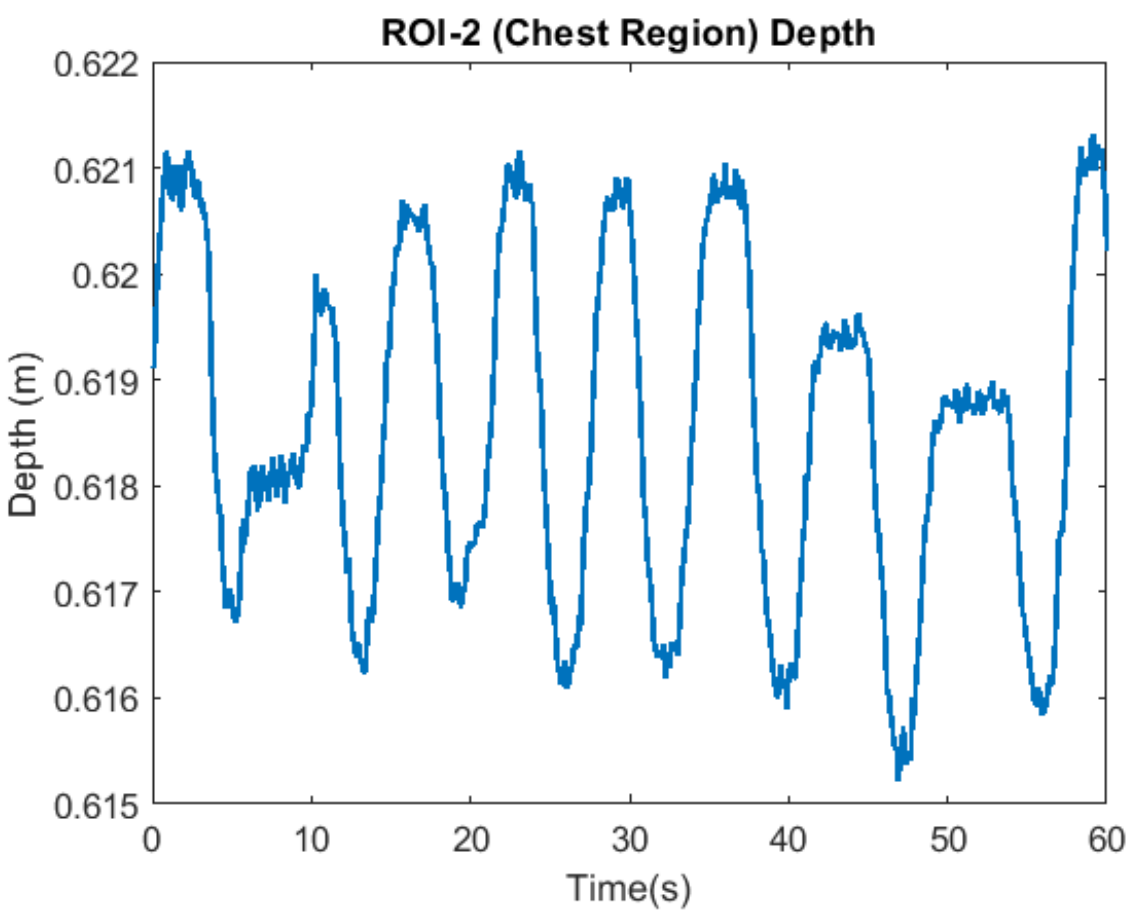

Figure 9. ROI-2 (chest) region depth signal, showing the periodic chest wall movement from respiration. The amplitude of the chest wall movement is $4 \mathrm{~mm}$.
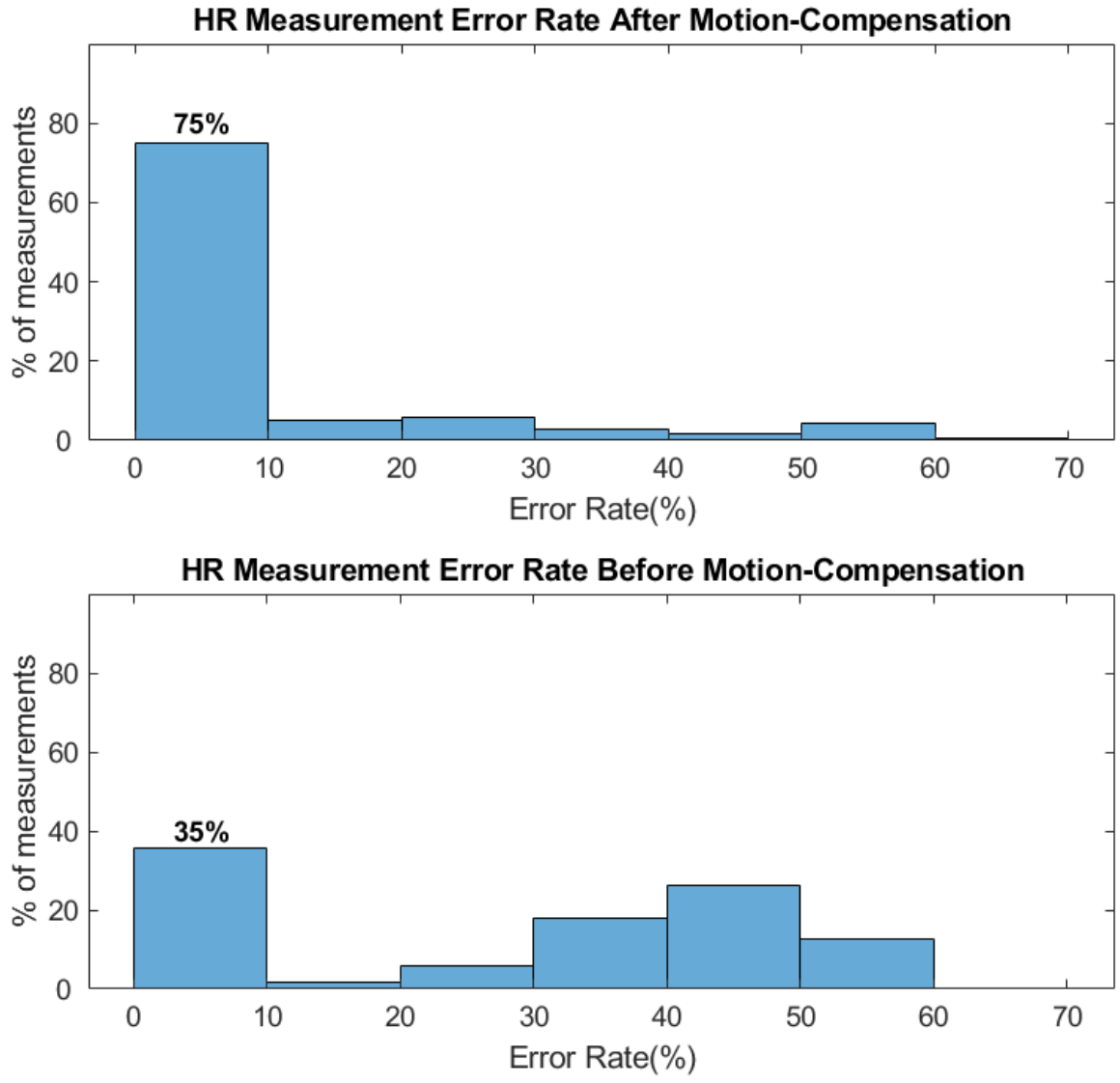

Figure 10. HR measurements error rate on all 286 measurements with motion compensation (top) and without motion compensation (bottom). 
To analyze the agreement between the reference device (finger pulse oximeter) against our system, we plot the Bland-Altman plot for the HR from the 29 participant study in Figure 11. The left plot illustrates the results with motion compensation, and the right plot illustrates the results without using motion compensation. The $x$-axis is the average HR reading of the reference oximeter and our ToF-based system, while the $y$-axis is the deviation of the HR measured in the ToF system from the reference oximeter reading. With compensation, the mean value of the deviation is $-6.25 \mathrm{BPM}$, and $95 \%$ of the deviation falls within $+/-35 \mathrm{BPM}$ of the mean deviation. In contrast, without compensation the mean value of the deviation is $21 \mathrm{BPM}$, and $95 \%$ of the deviation falls within $+/-36 \mathrm{BPM}$ of the mean deviation. The large standard deviation rises from the extreme erroneous values of the measurement system. These extreme values rise from camera noises as well as intensity artifacts that are not reflected in the depth signal. After the dominating motion artifacts are removed, the system could wrongly pick up these artifacts as HR. This also explains why the wrong measurements of the uncompensated signal groups around the top left corner of the plot since the dominating motion artifacts are typically at a lower frequency compared to HR. Additionally, because the motion artifacts are likely to be mistakenly picked as HR, it also leads to the seemingly small standard deviation of errors for the uncompensated HR measurements.
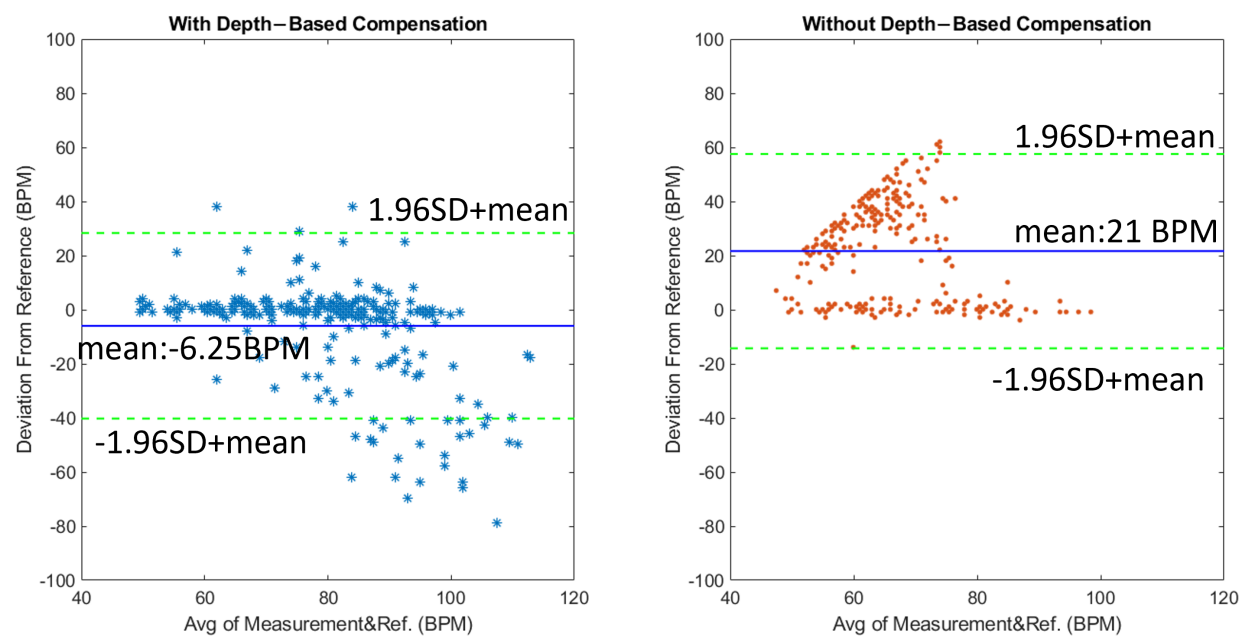

Figure 11. Bland-Altman plot for HR measurements on all 286 measurements with motion compensation (left) and without motion compensation (right).

The RR measurements are also examined for the pilot study of 29 participants. Figure 12 shows the percentage of 286 ToF-based RR measurements that fall into different bins of deviation from the reference Masimo pulse oximeter. The mean deviation from the reference reading is found to be $1.7 \mathrm{BPM}$ while $60 \%$ of measurements are within +/ - 2 BPM from the reference RR measurements. However, in our measurements, we can also sometimes see deviation as large as 10 BPM, these errors can be attributed to large body motion as well as the loose clothing that the participants are wearing. Some examples will be discussed in the discussion section. 


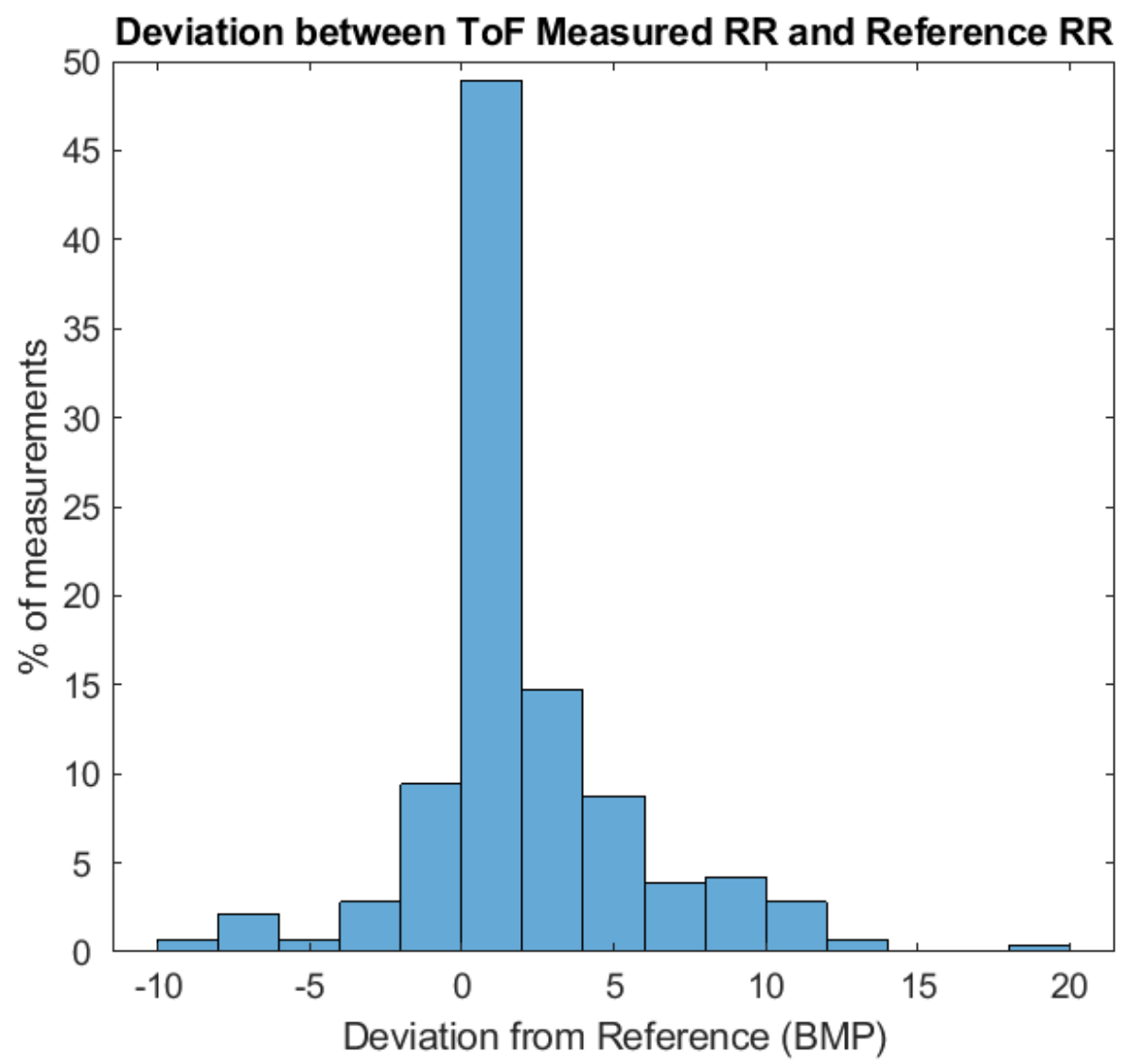

Figure 12. Percentage of ToF-based RR measurements versus deviation from reference oximeter.

\subsection{HR Measurement vs. Motion Artifacts}

To compare the performance between distance compensated and non-distance compensated HR measurements against different levels of motion, we plot the percentage of correct measurements versus a motion score (Figure 13). The blue bars are for the HR data with compensation, while the red bars are for the HR data without depth information compensation. In this study, we define motion score as the standard deviation of the mean depth change of the ROI- 1 to the optical center of the camera, where depth change is calculated as the difference between depth $D_{\text {raw }}$ of ROI-1 and the mean depth of ROI-1 over the 60 s measurement.

$$
\text { MotionScore }=\operatorname{std}\left(\operatorname{Abs}\left(\left(\operatorname{depth}_{\mathrm{ROI}-1}\right)-\operatorname{mean}\left(\operatorname{depth}_{\mathrm{ROI}-1}\right)\right)\right.
$$

Since the motion in real-life situations can sometimes be of an irregular pattern, the motion score defined in this study reflects amplitude and frequency of a participant's motion. The higher the motion score, the larger and more frequent the motion artifacts are. For example, a motion score below $0.3 \mathrm{~cm}$ is typical of a participant sitting still, while a motion score above $1 \mathrm{~cm}$ is typical of participants adjusting for a comfortable position e.g., sitting restlessly while sitting. It is worth noticing that a motion score of $1 \mathrm{~cm}$ does not necessarily mean the participant moves only $1 \mathrm{~cm}$ during the measurements. For example, in a measurement with a motion score of $1 \mathrm{~cm}$, the maximum ROI- 1 deviation from the mean position can exceed $4 \mathrm{~cm}$. The blue bars are for the HR measurements with compensation, while the red bars are for the HR measurements without depth information compensation. As can be seen from the plot, the performance of the motion-compensated HR measurements outperforms the uncompensated measurements across every motion score category. Even when small motion is introduced (motion score from 0 to $0.3 \mathrm{~cm}$ ), the distancecompensated HR measurement outperforms the uncompensated measurement by more 
than $75 \%$ in terms of success rate. Once the motion becomes larger, the performance of the uncompensated HR measurement plummets, indicating that the raw signal is dominated by motion artifacts. At the same time, the success rate of the motion-compensated HR also drops, but to a lesser extent. For example, when motion score exceeds $0.9 \mathrm{~cm}, \sim 35 \%$ of compensated measurements are correct meanwhile close to none of the uncompensated HR measurements are correct. This is of great significance because motion artifacts are a dominant source of noise in contactless HR measurement, especially with near-infrared wavelength where the absorption of blood is much weaker. We believe the performance of the ToF-based HR measurement system under larger motion could be improved with future development of both the motion compensation model as well as higher performance ToF cameras. We will discuss such future developments in more detail in the Section 5.

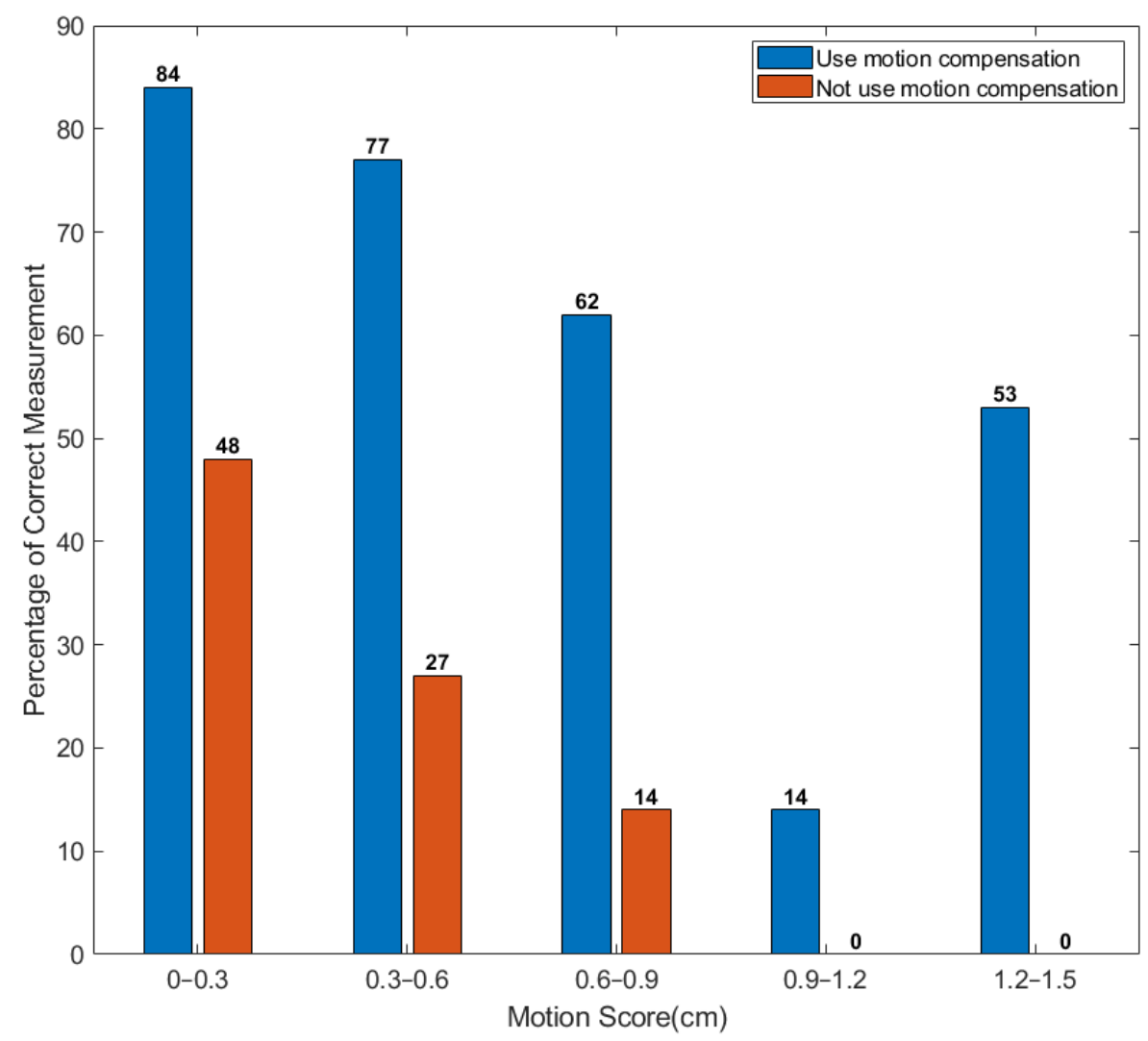

Figure 13. Percentage of correct HR measurement within each motion score category.

\subsection{Different Skin Color}

One of the challenges for an RGB-based HR/RR monitoring system is that different skin colors could affect the signal quality because of strong melanin absorption in the visible wavelength range [18]. In contrast, the absorption of melanin is much lower at near-infrared, which allows the ToF-based system to perform more uniformly among demographics of different skin colors. Figure 14 shows the error rate of the HR measurements among participants with darker skin color and lighter skin color. Participants with Fitzpatrick skin type I-III (17/29 participants) are considered as light skin color, while participants with skin type IV-VI (12/29 participants) are considered as darker skin color. Among the lighter skin color group, the percentage of success rate measurement is $74 \%$ with the mean deviation of the measurements being -6.1 BPM. As for the darker skin color group, the same success rate of $74 \%$ is achieved with the mean deviation from the reference at $-5.8 \mathrm{BPM}$. 

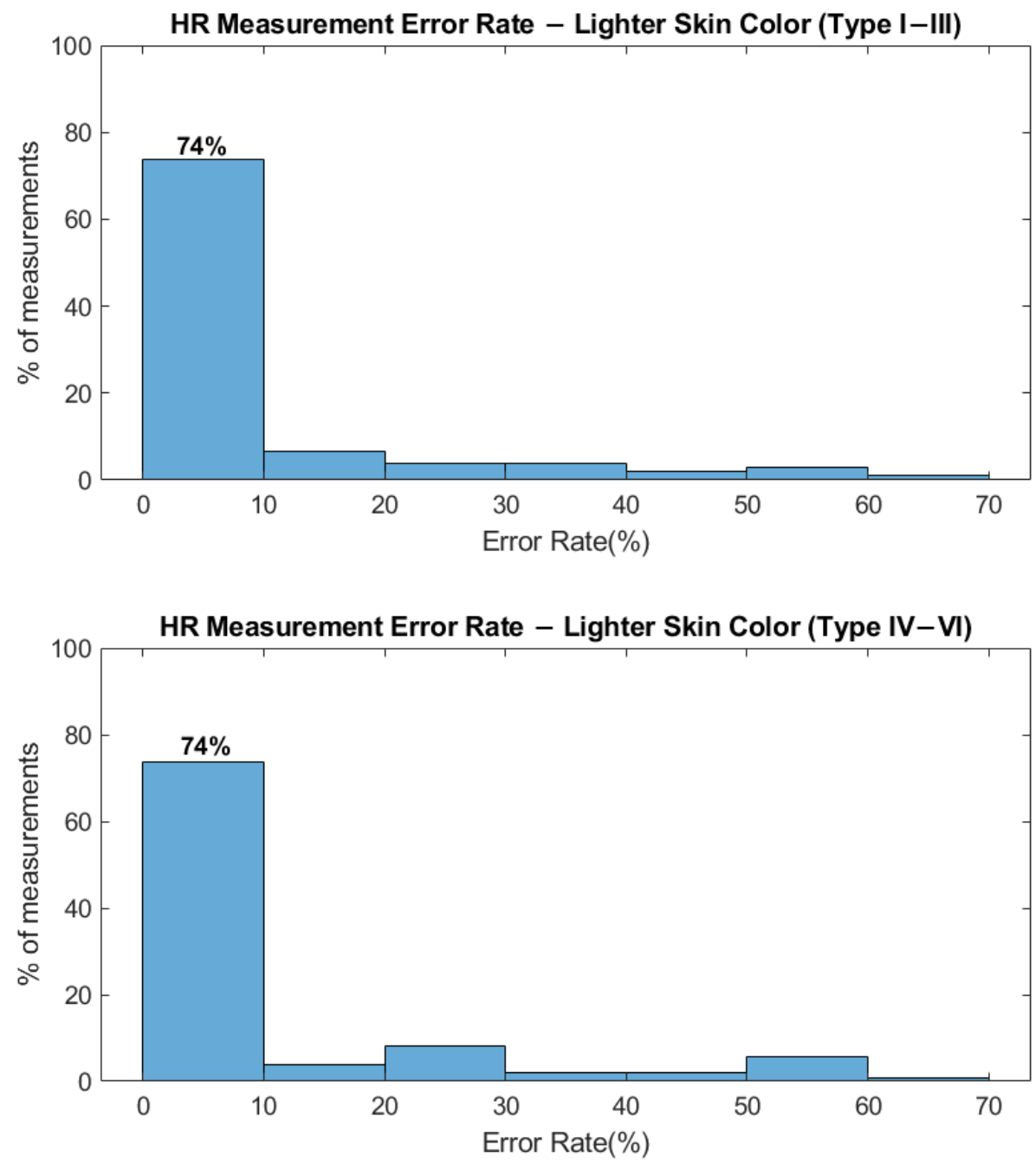

Figure 14. HR measurements error rate within groups of: lighter skin color (top) and darker skin color (bottom).

Similar performance among two skin color groups is also evident when we examine the Bland-Altman plot in Figure 15. Both the mean deviation (-6.1 BPM for the lighter skin color versus $-5.8 \mathrm{BPM}$ for darker skin color) and the $95 \%$ percentile range $(+/-35 \mathrm{BPM}$ for the lighter skin color versus +/ - 33 BPM for darker skin color) are very similar between the two groups. Moreover, deviation distribution is also similar among the two groups, indicating that the ToF system used in this study performs equally well among participants of different skin colors. Thus, using near-infrared illumination, we are able to achieve performance independent of skin color.

\subsection{Different Gender and Age}

We also investigate the performance of the ToF-based HR measurements system in terms of the participant's gender and age. Similar to the RGB camera-based system, no significant performance difference is seen among participants of different gender and age. Figure 16 shows the success percentage rate among participants of different gender, we achieve a $77 \%$ success rate in the male group and a $72 \%$ success rate in the female group. The mean HR deviation from reference is $-5.6 \mathrm{BPM}$ for the male group (18/29 participants) and -7.2 BPM for the female group (11/29 participants) (Figure 17), respectively. Even though the mean deviation differs slightly, the pattern of deviation data points looks similar. Such differences may be attributed to different skin conditions or even different skincare products used on the skin. Even though participants are asked not to use makeup, there could still be residuals left on the participant's face. Since the blood absorption at 
infrared is already low, any further decrease of signal could affect the success rate of the HR measurements.
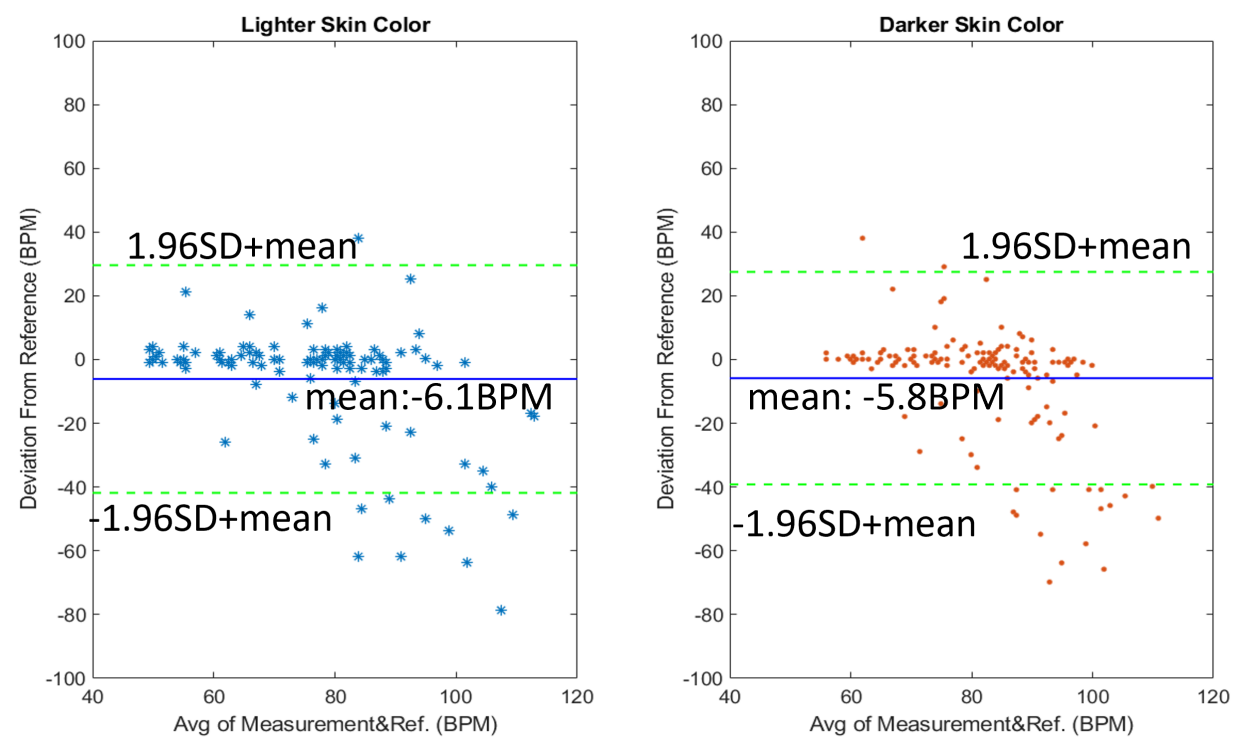

Figure 15. Bland-Altman plot for HR measurements within groups of: lighter skin color (left) and darker skin color (right).
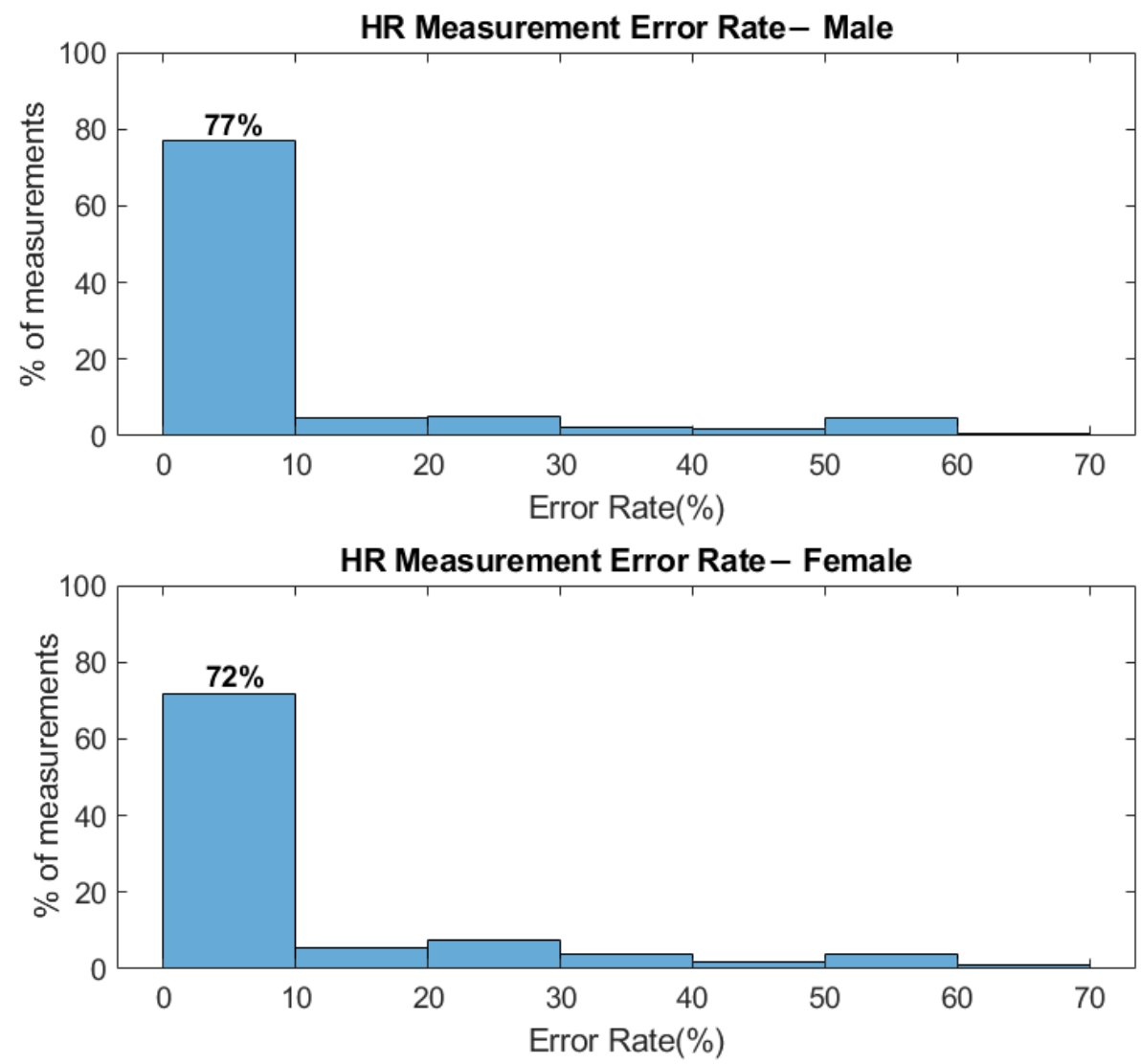

Figure 16. HR measurements error rate within groups of: male (top) and female (bottom).

In terms of age, we separated the participants into a younger group (21/29 participants) and an elder group (8/29 participants) at the threshold of 35 years old. The success rate/mean deviation is $84 \% /-7.7 \mathrm{BPM}$ for the elder group and 71\%/-5.89 $\mathrm{BPM}$ for the younger group (Figures 18 and 19). The higher success rate among the elder participants 
could be caused by elder participants staying more still during the measurements compared to younger participants. In fact, the mean motion score for the younger group is $0.61 \mathrm{~cm}$, compared to $0.47 \mathrm{~cm}$ for the elder groups. As we discussed before, the success rate deteriorates as the motion score increases, which could explain the higher success rate among elder participants.
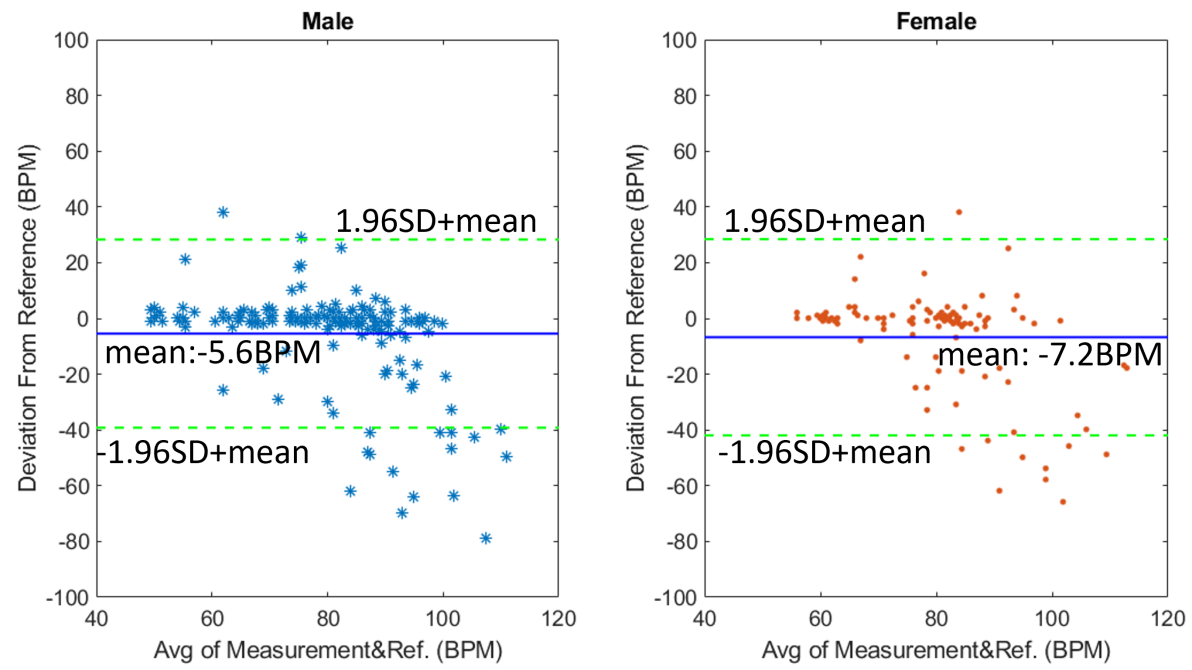

Figure 17. Bland-Altman plot for HR measurements within groups of: male (left) and female (right).
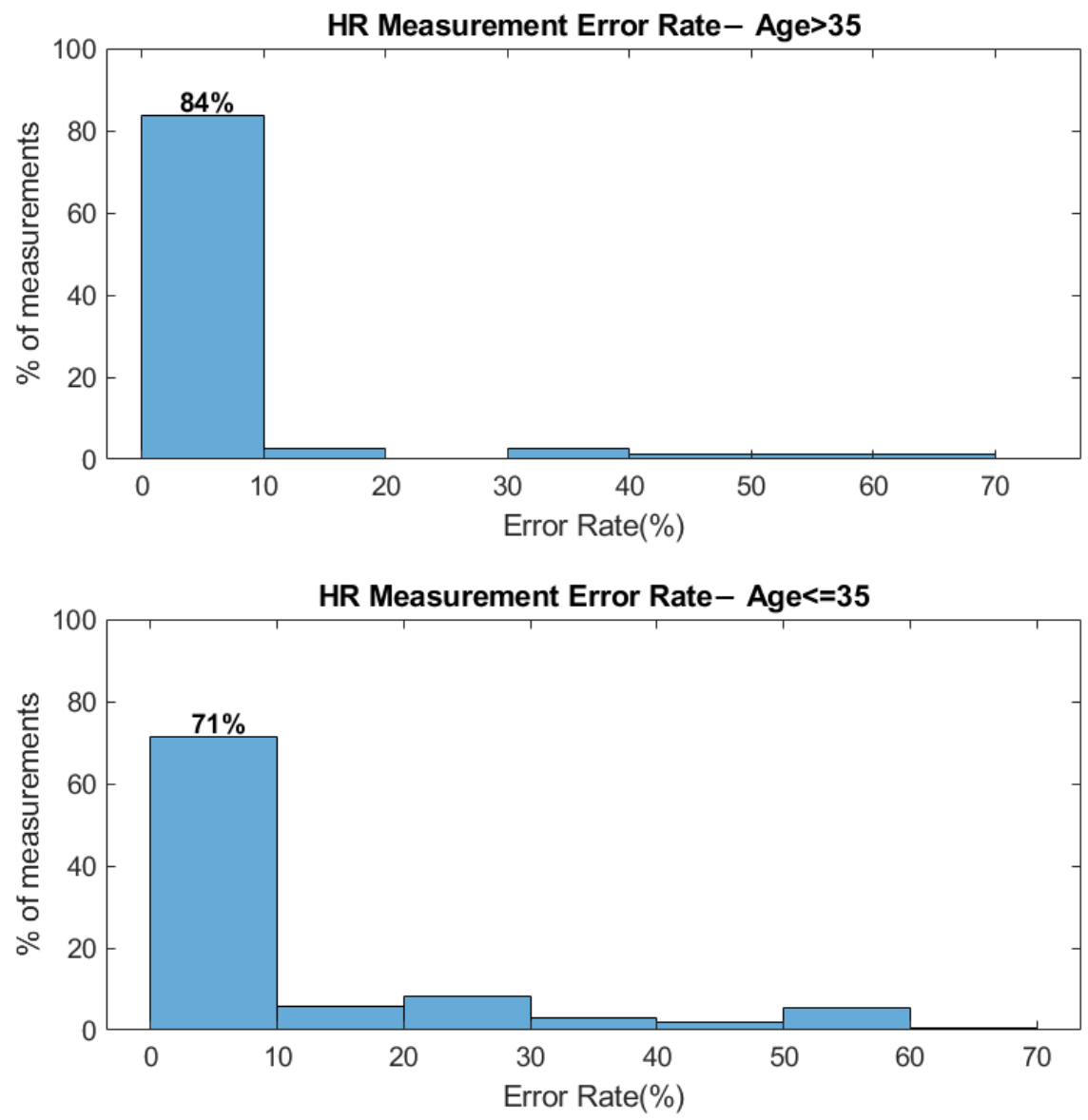

Figure 18. HR measurements error rate within groups of: age $>35$ (top) and age $<=35$ (bottom). 

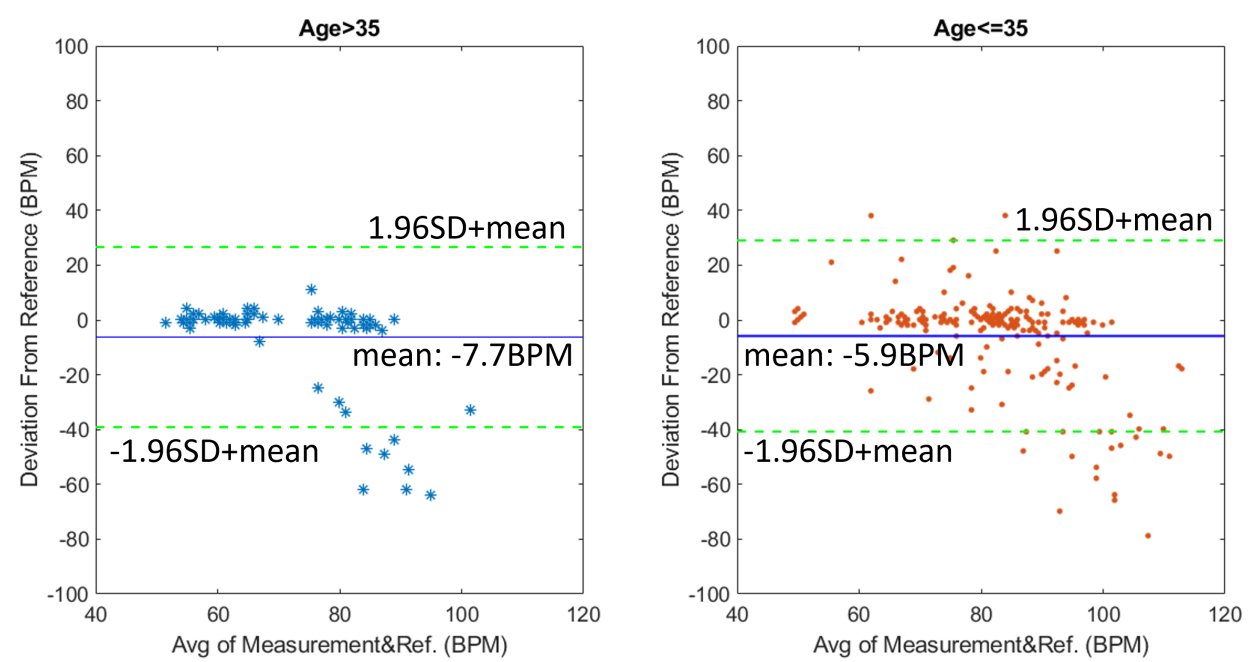

Figure 19. Bland-Altman plot for HR measurements within groups of: age $>35$ (left) and age $<=35$ (right).

\section{Discussion}

Even though the additional depth information from the ToF-based HR/RR measurements system shows benefits in terms of motion compensation and respiration rate measurements, we also noticed several external factors that can affect the performance, which could help with the future improvement of the system's performance if addressed.

\subsection{Limitation of RR Measurements}

In this study, the RRs are acquired by directly measuring the displacement of the chest wall during respiration. In reality, it is often the case when the users' bodies are involuntarily moving while the system is measuring the respiration rate. Therefore, similar to the HR measurement, the RR measurement could also suffer from motion-induced artifacts. Even though such an issue is less pronounced in this study as the participants are asked to sit in front of the camera, we still discuss possible methods to mitigate the effects of body motion that is unrelated to respiration. Since the neck region usually moves with the chest but does not show a strong respiration motion, we could use the depth signal on the neck as a reference region to separate the respiration motion from other body motions. Therefore, we could view the depth signal from the chest region as a superposition of the general body motion and the chest wall motion from respiration. A blind source separation method such as Independent Component Analysis (ICA) can then be applied to the raw depth signals to extract the respiration motion. For example, Figure 20a shows the raw depth signal from the neck and chest region, respectively. The depth signal from the neck region records mainly the motion of the body since respiration does not cause significant muscle contraction on the neck. As a comparison, the signal from the chest region (Figure 20a bottom figure) is also affected by the overall body motion as the periodic chest wall motion from respiration is masked. The signal after ICA separation is shown in 1Figure 20b. A clear pattern of the chest wall moving back and forth from the respiring motion can now be seen in the top row of the separated signal. The respiratory rate, derived from the first component in Figure 9, is measured as 15 BPM, while the reference pulse oximeter also measures $15 \mathrm{BPM}$.

It is also worth noticing that since the method of acquiring the respiration rate relies on directly measuring chest wall movement, any type of clothing, such as a loose sweatshirt or down jacket, that obfuscates the chest wall movement, will prevent the system from acquiring RR. To overcome this limitation, some complementary methods such as deriving RR from HR variability or using a thermal sensor to measure respiration rate can be used in combination with the ToF camera to provide RR measurements in more versatile environments $[5,7,26,27]$. 

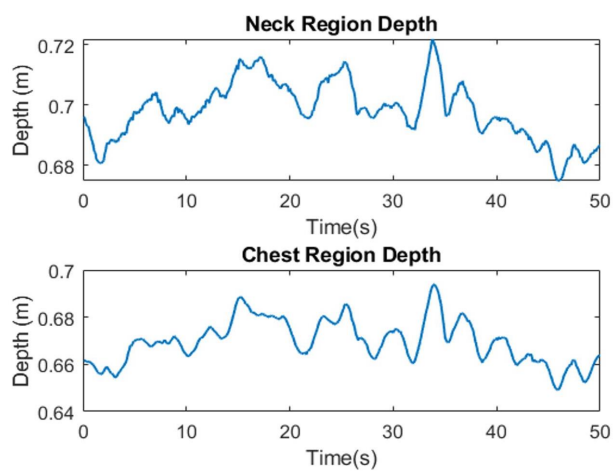

(a)

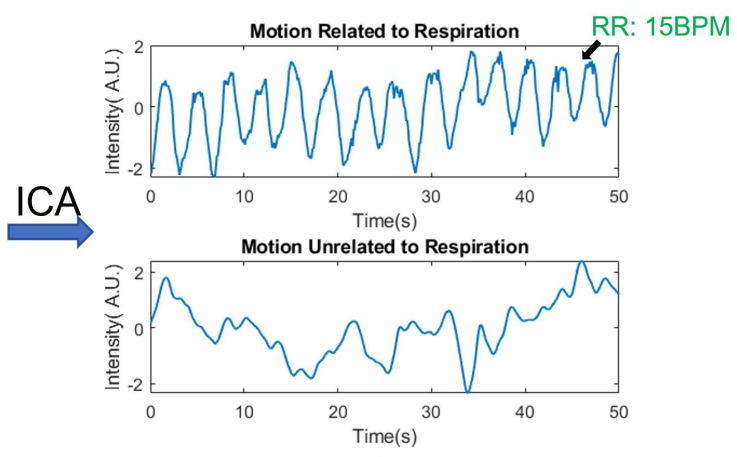

(b)

Figure 20. (a) Time domain signal for RR extraction: Raw depth signal from neck region not containing RR motion (top), raw depth signal from chest region containing RR motion (bottom). (b) Depth signal after ICA separation: Signal component with RR motion (top), signal component without RR motion compensation (bottom).

\subsection{Limitation of Motion Compensated HR Measurement Using ToF Camera}

The results of the pilot human study suggest that the magnitude of motion contributes the most to the failure of the HR measurements. When the motion score is relatively small $(<0.3 \mathrm{~cm})$, the success rate is $84 \%$ while such success rate falls quickly as the motion score increases. The adverse effects of larger motion to the HR measurements seem intuitive as it both makes the ROIs harder to track as well as creates larger intensity artifacts for the ToF camera to compensate. However, there are also some less noticeable factors that can also contribute to the erroneous reading even when the motion artifacts are small.

To obtain a stable signal from a ROI, the ROIs needs to be stably and accurately tracked in every frame. In some cases, reflective spots on the face as well as the changing head pose/facial expression could introduce artifacts to the tracked ROI. For example, we find that the light reflection from the participants' eyes combined with the blinking motion can sometimes cause the positions of the ROI to jitter, even though the participants are not moving at that time. If such artifacts are present during measurement, it can mislead the system to take the blinking frequency as HR.

In terms of the limitation of the depth-based motion compensation, other than the obvious cases where significant motion overwhelms the underlying HR signal, we also find the system performance degrading when local motions, such as involuntary muscle twitches or facial expression changes $[6,10]$, are present. Since the human face is not a rigid body, these local motions can cause intensity artifacts without inducing a change in depth signal at the same time, making them "invisible" to the depth-based motion compensation.

Therefore, the depth signal-based method is more effective toward motion artifacts that induce depth variations that are large enough to be captured by the ToF camera. As an example, global motions, especially scaling and translational motion, can be easily captured by the ToF camera. Even though these larger motions cause large grayscale intensity change on the ToF camera, they can be effectively removed to prevent them from affecting HR measurement.

\subsection{Future Improvements}

Based on the previous discussion, we believe that the system performance can be further improved by building a more precise model to compensate for motion-induced artifacts and by developing ToF cameras with higher resolution and frame rate. The model we use in this study to compensate for the intensity change caused by motion is relatively coarse as we only consider the average depth change within a larger ROI. If a model can be developed to further utilize the point cloud generated by the ToF camera to take more subtle movements such as head rotation/tilting into consideration, it would expand the types of motion artifacts that the CVSM system can compensate. At the same time, efforts are made to develop an indirect ToF camera with a higher resolution and frame rate [39]. 
Higher camera resolution could be useful in improving the ROI tracking performance of the system while a higher frame rate would allow the system to capture the motion of higher frequency without aliasing or motion blur. In addition, to compensate for micro-motion that is not captured by the depth signal, other signal processing techniques such as sparse frequency estimation could be combined with our depth-based motion compensation method to improve the motion artifacts resilience of the system.

\section{Conclusions}

In conclusion, we demonstrate a contactless HR/RR monitoring system based on an indirect ToF camera. The active illumination from an $850 \mathrm{~nm}$ VCSEL combined with the depth information from the ToF camera can be used to compensate for the motion artifacts that commonly dominate the underlying heartbeat signal. We show that the depth-based compensation increases success rate (defined as the HR reading within $10 \%$ of the reference HR measurement) to $75 \%$, as compared to $35 \%$ when no depth compensation method is used. The additional depth information also enables the direct measurement of RR from a participant by measuring the chest wall movement during breathing. The RR measurement from our system shows a mean deviation of 1.7 BPM compared to the reference RR reading. Furthermore, the infrared active illumination makes this system less affected by factors such as environmental lighting or the skin color of the user. In our pilot study with 29 participants, we show that our system performs similarly among participants of different demographics such as skin color, age, and gender. In the future, this system could be found useful in fields such as in-cabin driver monitoring and remote patient monitoring where non-contact unobtrusive and environmentally agnostic physiological monitoring is preferred $[19,23,40,41]$.

Author Contributions: Conceptualization, K.G., M.N.I. and T.Z.; methodology, K.G., T.Z. and C.J.V.; software, K.G., T.Z. and A.A.; validation, K.G. and E.P.; data analysis, K.G.; data curation, K.G., C.J.V. and E.P.; writing—original draft preparation, K.G.; writing—review and editing, K.G., E.P. and M.N.I.; visualization, K.G.; supervision, M.N.I.; project administration, M.N.I.; funding acquisition, M.N.I. All authors have read and agreed to the published version of the manuscript.

Funding: This study received no external funding.

Institutional Review Board Statement: The study was conducted according to the guidelines of the Declaration of Helsinki, and approved by the Institutional Review Board of the University of Michigan (protocol code HUM00191000, 3 March 2021).

Informed Consent Statement: Informed consent was obtained from all subjects involved in the study.

Data Availability Statement: The data presented in this study are available on request from the corresponding author. The data are not publicly available due to privacy concerns as well as regulation from the Institutional Review Board.

Acknowledgments: The authors of this study thank Ioulia Kolveman, Fred Terry, and Jenna Wiens for their support in data collection, study design, and data analysis.

Conflicts of Interest: Mohammed N. Islam is the founder and chief technology officer of Omni Science Inc.

\section{References}

1. Verkruysse, W.; Svaas, L.O.; Nelson, J.S. Remote plethysmographic imaging using ambient light. Opt. Express 2008, 16, 21434-21445. [CrossRef]

2. Huang, P.W.; Wu, B.J.; Wu, B.F. A heart rate monitoring framework for real-world drivers using remote photoplethysmography. IEEE J. Biomed. Health Inform. 2020, 25, 1397-1408. [CrossRef]

3. Balakrishnan, G.; Dur, F.; Guttag, J. Detecting pulse from head motions in video. In Proceedings of the IEEE Conference on Computer Vision and Pattern Recognition, Portland, OR, USA, 23-28 June 2013.

4. Cheng, J.; Chen, X.; Xu, L.; Wang, Z.J. Illumination variation-resistant video-based heart rate measurement using joint blind source separation and ensemble empirical mode decomposition. IEEE J. Biomed. Health Inform. 2016, 21, 1422-1433. [CrossRef] [PubMed] 
5. Negishi, T.; Abe, S.; Matsui, T.; Liu, H.; Kurosawa, M.; Kirimoto, T.; Sun, G. Contactless vital signs measurement system using RGB-thermal image sensors and its clinical screening test on patients with seasonal influenza. Sensors 2020, 20, 2171. [CrossRef]

6. Kumar, M.; Veeraraghavan, A.; Sabharwal, A. DistancePPG: Robust non-contact vital signs monitoring using a camera. Biomed. Opt. Express 2015, 6, 1565-1588. [CrossRef]

7. Sun, G.; Nakayama, Y.; Dagdanpurev, S.; Abe, S.; Nishimura, H.; Kirimoto, T.; Matsui, T. Remote sensing of multiple vital signs using a CMOS camera-equipped infrared thermography system and its clinical application in rapidly screening patients with suspected infectious diseases. Int. J. Infect. Dis. 2017, 55, 113-117. [CrossRef]

8. Unursaikhan, B.; Tanaka, N.; Sun, G.; Watanabe, S.; Yoshii, M.; Funahashi, K.; Sekimoto, F.; Hayashibara, F.; Yoshizawa, Y.; Choimaa, L.; et al. Development of a Novel Web Camera-Based Contact-Free Major Depressive Disorder Screening System Using Autonomic Nervous Responses Induced by a Mental Task and Its Clinical Application. Front. Physiol. 2021, 12, 706. [CrossRef] [PubMed]

9. Wang, W.; den Brinker, A.C.; Stuijk, S.; De Haan, G. Algorithmic principles of remote PPG. IEEE Trans. Biomed. Eng. 2016, 64, 1479-1491. [CrossRef]

10. Wang, W.; Stuijk, S.; De Haan, G. Exploiting spatial redundancy of image sensor for motion robust rPPG. IEEE Trans. Biomed. Eng. 2014, 62, 415-425. [CrossRef]

11. de Haan, G.; Jeanne, V. Robust pulse rate from chrominance-based rPPG. IEEE Trans. Biomed. Eng. 2013, 60, 2878-2886. [CrossRef] [PubMed]

12. Wang, W.; Stuijk, S.; De Haan, G. A novel algorithm for remote photoplethysmography: Spatial subspace rotation. IEEE Trans. Biomed. Eng. 2016, 63, 1974-1984. [CrossRef] [PubMed]

13. De Haan, G.; Van Leest, A. Improved motion robustness of remote-PPG by using the blood volume pulse signature. Physiol. Meas. 2014, 35, 1913. [CrossRef]

14. Wu, B.F.; Huang, P.W.; Lin, C.H.; Chung, M.L.; Tsou, T.Y.; Wu, Y.L. Motion resistant image-photoplethysmography based on spectral peak tracking algorithm. IEEE Access 2018, 6, 21621-21634. [CrossRef]

15. Feng, L.; Po, L.M.; Xu, X.; Li, Y.; Ma, R. Motion-resistant remote imaging photoplethysmography based on the optical properties of skin. IEEE Trans. Circuits Syst. Video Technol. 2014, 25, 879-891. [CrossRef]

16. Aoxin, N.; Azarang, A.; Kehtarnavaz, N. A Review of Deep Learning-Based Contactless Heart Rate Measurement Methods. Sensors 2021, 21, 3719.

17. Zaunseder, S.; Trumpp, A.; Wedekind, D.; Malberg, H. Cardiovascular assessment by imaging photoplethysmography-A review. Biomed. Eng. Tech. 2018, 63, 617-634. [CrossRef] [PubMed]

18. Zonios, G.; Dimou, A.; Bassukas, I.; Galaris, D.; Tsolakidis, A.; Kaxiras, E. Melanin absorption spectroscopy: New method for noninvasive skin investigation and melanoma detection. J. Biomed. Opt. 2008, 13, 014017. [CrossRef] [PubMed]

19. Gastel, V.; Stuijk, M.S.; de Haan, G. Motion robust remote-PPG in infrared. IEEE Trans. Biomed. Eng. 2015, 62, 1425-1433. [CrossRef]

20. Yu, S.; Hu, S.; Azorin-Peris, V.; Chambers, J.A.; Zhu, Y.; Greenwald, S.E. Motion-compensated noncontact imaging photoplethysmography to monitor cardiorespiratory status during exercise. J. Biomed. Opt. 2011, 16, 077010.

21. Magdalena, N.E.; Marks, T.K.; Mansour, H.; Veeraraghavan, A. SparsePPG: Towards driver monitoring using camera-based vital signs estimation in near-infrared. In Proceedings of the IEEE Conference on Computer Vision and Pattern Recognition Workshops, Salt Lake City, UT, USA, 18-22 June 2018.

22. Perdue, K.L.; Westerlund, A.; McCormick, S.A.; Nelson, C.A., III. Extraction of heart rate from functional near-infrared spectroscopy in infants. J. Biomed. Opt. 2014, 19, 067010. [CrossRef]

23. Blanik, N.; Heimann, K.; Pereira, C.; Paul, M.; Blazek, V.; Venema, B.; Orlikowsky, T.; Leonhardt, S. Remote vital parameter monitoring in neonatology-robust, unobtrusive heart rate detection in a realistic clinical scenario. Biomed. Eng. Tech. 2016, 61, 631-643. [CrossRef] [PubMed]

24. Nahler, C.; Feldhofer, B.; Ruether, M.; Holweg, G.; Druml, N. Exploring the usage of Time-of-Flight Cameras for contact and remote Photoplethysmography. In Proceedings of the 2018 21st Euromicro Conference on Digital System Design (DSD), Prague, Czech Republic, 29-31 August 2018.

25. Kranjec, J.; Beguš, S.; Geršak, G.; Drnovšek, J. Non-contact heart rate and heart rate variability measurements: A review. Biomed. Signal Process. Control 2014, 13, 102-112. [CrossRef]

26. van Gastel, M.; Stuijk, S.; de Haan, G. Robust respiration detection from remote photoplethysmography. Biomed. Opt. Express 2016, 7, 4941-4957. [CrossRef] [PubMed]

27. Charlton, P.H.; Bonnici, T.; Tarassenko, L.; Clifton, D.A.; Beale, R.; Watkinson, P.J. An assessment of algorithms to estimate respiratory rate from the electrocardiogram and photoplethysmogram. Physiol. Meas. 2016, 37, 610-626. [CrossRef] [PubMed]

28. Bernacchia, N.; Scalise, L.; Casacanditella, L.; Ercoli, I.; Marchionni, P.; Tomasini, E.P. Non contact measurement of heart and respiration rates based on Kinect ${ }^{\mathrm{TM}}$. In Proceedings of the 2014 IEEE International Symposium on Medical Measurements and Applications (MeMeA), Lisboa, Portugal, 11-12 June 2014.

29. Procházka, A.; Schätz, M.; Vyšata, O.; Vališ, M. Microsoft kinect visual and depth sensors for breathing and heart rate analysis. Sensors 2016, 16, 996. [CrossRef]

30. Zanuttigh, P.; Marin, G.; Dal Mutto, C.; Dominio, F.; Minto, L.; Cortelazzo, G.M. Time-of-Flight and Structured Light Depth Cameras; Technology and Applications; Springer: Cham, Switzerland, 2016. 
31. Martinez, L.F.; Paez, G.; Strojnik, M. Optimal wavelength selection for noncontact reflection photoplethysmography. In Proceedings of the 22nd Congress of the International Commission for Optics: Light for the Development of the World, Puebla, Mexico, 15-19 August 2011; International Society for Optics and Photonics: Bellingham, WA, USA, 2011; Volume 8011.

32. Moço, A.V.; Stuijk, S.; de Haan, G. New insights into the origin of remote PPG signals in visible light and infrared. Sci. Rep. 2018, 8,1-15. [CrossRef]

33. Asthana, A.; Zafeiriou, S.; Cheng, S.; Pantic, M. Incremental face alignment in the wild. In Proceedings of the IEEE Conference on Computer Vision and Pattern Recognition, Columbus, OH, USA, 23-28 June 2014.

34. Zhu, X.; Ramanan, D. Face detection, pose estimation, and landmark localization in the wild. In Proceedings of the 2012 IEEE Conference on Computer Vision and Pattern Recognition, Providence, RI, USA, 16-21 June 2012.

35. So-Ling, C.; Li, L. A multi-layered reflection model of natural human skin. In Proceedings of the Computer Graphics International 2001, Hong Kong, China, 6 July 2001.

36. Weyrich, T.; Matusik, W.; Pfister, H.; Bickel, B.; Donner, C.; Tu, C.; McAndless, J.; Lee, J.; Ngan, A.; Jensen, H.W.; et al. Analysis of human faces using a measurement-based skin reflectance model. ACM Trans. Graph. (ToG) 2006, 25, 1013-1024. [CrossRef]

37. Shi, J. Good features to track. In Proceedings of the IEEE Conference on Computer Vision and Pattern Recognition, Seattle, WA, USA, 21-23 June 1994.

38. Fitzpatrick, T.B. The validity and practicality of sun-reactive skin types I through VI. Arch. Dermatol. 1988, 124, 869-871. [CrossRef]

39. Keel, M.S.; Kim, D.; Kim, Y.; Bae, M.; Ki, M.; Chung, B.; Son, S.; Lee, H.; Jo, H.; Shin, S.C.; et al. A 4-tap $3.5 \mu \mathrm{m} 1.2$ Mpixel Indirect Time-of-Flight CMOS Image Sensor with Peak Current Mitigation and Multi-User Interference Cancellation. In Proceedings of the 2021 IEEE International Solid-State Circuits Conference (ISSCC), San Francisco, CA, USA, 13-22 February 2021 ; Volume 64.

40. Wang, J.; Warnecke, J.M.; Haghi, M.; Deserno, T.M. Unobtrusive health monitoring in private spaces: The smart vehicle. Sensors 2020, 20, 2442. [CrossRef]

41. Jiang, C.; Yabroff, K.R.; Deng, L.; Perimbeti, S.; Han, X. Prevalence of Underlying Medical Conditions Associated with Severe COVID-19 Illness in Adult Cancer Survivors in the United States. JNCI J. Natl. Cancer Inst. 2021, djab012. [CrossRef] 